\title{
Synthesis of Novel Polyyne Analogues of Sphingoid Base via an Iterative Acetylene Homologation Sequence
}

Sanghee Kim,* Yun Mi Lee, Hee Ryong Kang, Jihee Cho, Taeho Lee, and Deukjoon Kim

\section{Contents}

General Experimental Method

Full experimental procedures and analytical data of compounds

${ }^{1} \mathrm{H}$ NMR and ${ }^{13} \mathrm{C}$ NMR spectra of compound $\mathbf{1 2}$

S15-S16

${ }^{1} \mathrm{H}$ NMR and ${ }^{13} \mathrm{C}$ NMR spectra of compound $\mathbf{3 a}$

S17-S18

${ }^{1} \mathrm{H}$ NMR and ${ }^{13} \mathrm{C}$ NMR spectra of compound $\mathbf{2 a}$

S19-S20

${ }^{1} \mathrm{H}$ NMR and ${ }^{13} \mathrm{C}$ NMR spectra of compound $\mathbf{1 6 a}$

$\mathrm{S} 21-\mathrm{S} 22$

${ }^{1} \mathrm{H}$ NMR and ${ }^{13} \mathrm{C}$ NMR spectra of compound $\mathbf{1 6 b}$

S23-S24

${ }^{1} \mathrm{H}$ NMR and ${ }^{13} \mathrm{C}$ NMR spectra of compound $\mathbf{3 b}$

S25-S26

${ }^{1} \mathrm{H}$ NMR and ${ }^{13} \mathrm{C}$ NMR spectra of compound $\mathbf{3 c}$

S27-S28

${ }^{1} \mathrm{H}$ NMR and ${ }^{13} \mathrm{C}$ NMR spectra of compound $\mathbf{2 b}$

S29-S30

${ }^{1} \mathrm{H}$ NMR and ${ }^{13} \mathrm{C}$ NMR spectra of compound 2c

S31-S32 
General. All chemicals were reagent grade and used as purchased. All reactions were performed under an inert atmosphere of dry argon or nitrogen using distilled dry solvents. Reactions were monitored by TLC analysis using silica gel 60 F-254 TLC plates. Melting points are uncorrected. Flash column chromatography was carried out on silica gel (230-400 mesh). Optical rotations were measured using sodium light (D line $589.3 \mathrm{~nm}) .{ }^{1} \mathrm{H}$ NMR and ${ }^{13} \mathrm{C}$ NMR spectra were recorded in $\delta$ units relative to deuterated solvent as internal reference at 300 and $75 \mathrm{MHz}$, respectively. IR spectra were measured on Fourier Transform Infrared spectrometer. High resolution mass spectra (HRMS) were recorded using electron impact (EI), fast atom bombardment (FAB), or chemical ionization (CI). 


\section{(S)-tert-Butyl 4-[(R)-1-hydroxy-3-(triisopropylsilyl)prop-2-ynyl[-2,2-}

dimethyloxazolidine-3-carboxylate (10).

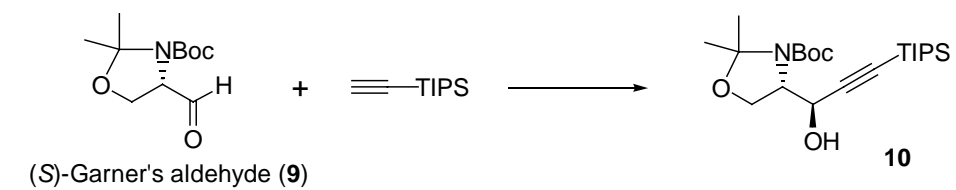

To a solution of (triisopropyl)acetylene $(5.90 \mathrm{~mL}, 26.3 \mathrm{mmol})$ in toluene $(16 \mathrm{~mL})$ and HMPA $(5 \mathrm{~mL})$ was slowly added $n$-BuLi $(16.0 \mathrm{~mL}, 25.6 \mathrm{mmol}, 1.6 \mathrm{M}$ solution in hexane) at $-78{ }^{\circ} \mathrm{C}$. After stirring for $30 \mathrm{~min}$ at this temperature, a solution of $(S)$ Garner's aldehyde (9) $(3.00 \mathrm{~g}, 13.1 \mathrm{mmol})$ in toluene $(10 \mathrm{~mL})$ was slowly added over $10 \mathrm{~min}$ at $-78{ }^{\circ} \mathrm{C}$. After stirring for $30 \mathrm{~min}$ at $-78{ }^{\circ} \mathrm{C}$, the reaction mixture was slowly warmed to room temperature and quenched with $1 \mathrm{M} \mathrm{NaH} \mathrm{NO}_{4}$ solution. It was diluted with EtOAc and washed with brine. The organic layer was dried over $\mathrm{MgSO}_{4}$ and concentrated. The residue was purified by silica gel column chromatography (hexane/EtOAc, 8:1) to give the desired erythro-isomer $\mathbf{1 0}(5.04 \mathrm{~g}, 93 \%)$ as a colorless oil. Crude ${ }^{1} \mathrm{H}$ NMR analysis showed traces of threo-isomer (erythro/threo, 21:1): $[\alpha]^{22}{ }_{D}$ $-34.7\left(\right.$ c $\left.2.5, \mathrm{CHCl}_{3}\right) ;{ }^{1} \mathrm{H} \mathrm{NMR}\left(300 \mathrm{MHz}, \mathrm{CDCl}_{3}\right.$, mixture of rotamers) $\delta 1.01(\mathrm{~s}, 21 \mathrm{H})$, 1.36- 1.59 (m, 15H), 3.87-4.05 (m, 2H), 4.48-4.62 (m, 2H); ${ }^{13} \mathrm{C}$ NMR (75 MHz, $\mathrm{CDCl}_{3}$, rotamer 1/rotamer 2) $\delta 11.0,18.43 / 18.44,25.9 / 25.3,28.2,62.3,63.3,64.7,81.0,86.7$, 94.9, 105.5, 153.7; HRMS (EI) calcd for $\mathrm{C}_{22} \mathrm{H}_{42} \mathrm{NO}_{4} \mathrm{Si}\left([\mathrm{M}+\mathrm{H}]^{+}\right)$412.2883, found 412.2888

(S)-tert-Butyl 4-[(R)-1-acetoxy-3-(triisopropylsilyl)prop-2-ynyl]-2,2dimethyloxazolidine-3-carboxylate (11). 


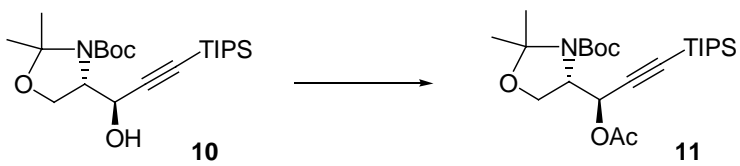

To a solution of $\mathbf{1 0}(1.20 \mathrm{~g}, 2.92 \mathrm{mmol})$ in pyridine $(10 \mathrm{~mL})$ were added acetic anhydride $(0.83 \mathrm{~mL}, 8.78 \mathrm{mmol})$ and DMAP $(17.8 \mathrm{mg}, 0.15 \mathrm{mmol})$. The reaction mixture was stirred at room temperature for $1 \mathrm{~h}$. Then, it was diluted with hexane and washed with water. The organic layer was dried over $\mathrm{MgSO}_{4}$, and concentrated. The residue was purified by silica gel column chromatography (hexane/EtOAc, 8:1) to give $11(1.28 \mathrm{~g}, 96 \%)$ as a colorless oil: $[\alpha]^{19}{ }_{\mathrm{D}}-90.9\left(c\right.$ 0.8, $\left.\mathrm{CHCl}_{3}\right) ;{ }^{1} \mathrm{H}$ NMR $(300 \mathrm{MHz}$, $\mathrm{CDCl}_{3}$, ca 6:4 mixture of rotamers) $\delta 0.99$ and 1.00 (each s, total $\left.21 \mathrm{H}\right), 1.40-1.49(\mathrm{~m}$, $15 \mathrm{H}), 2.04(\mathrm{~s}, 3 \mathrm{H}), 3.97-4.16(\mathrm{~m}, 2 \mathrm{H}), 4.23-4.30(\mathrm{~m}, 1 \mathrm{H}), 5.88(\mathrm{~d}, J=1.5 \mathrm{~Hz}, 0.6 \mathrm{H})$, $5.96(\mathrm{~d}, J=2.1 \mathrm{~Hz}, 0.4 \mathrm{H}) ;{ }^{13} \mathrm{C} \mathrm{NMR}\left(75 \mathrm{MHz}, \mathrm{CDCl}_{3}\right.$, rotamer 1/rotamer 2) $\delta 10.9$, $18.4,20.9,23.2 / 24.5,25.6 / 26.4,28.2,60.0 / 60.4,62.8 / 62.6,64.1 / 63.7,80.5 / 80.6$, 88.6/88.2, 94.9/94.2, 102.3, 151.3/152.2, 169.4/169.3; HRMS (CI) calcd for $\mathrm{C}_{24} \mathrm{H}_{44} \mathrm{NO}_{5} \mathrm{Si}\left([\mathrm{M}+\mathrm{H}]^{+}\right)$454.2988, found 454.2994.

\section{(S)-tert-Butyl 4-[(R)-1-acetoxy-3-bromoprop-2-ynyl]-2,2-dimethyloxazolidine-3-} carboxylate (12).

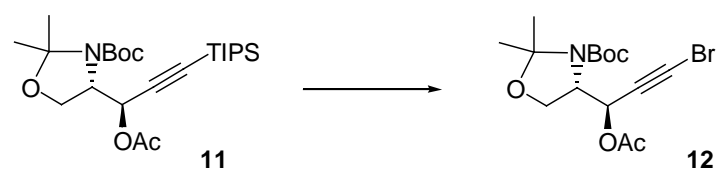

To a solution of $\mathbf{1 1}(1.80 \mathrm{~g}, 3.97 \mathrm{mmol})$ in acetonitrile $(40 \mathrm{~mL})$ were added NBS (848 $\mathrm{mg}, 4.76 \mathrm{mmol})$ and $\mathrm{AgF}(640 \mathrm{mg}, 5.04 \mathrm{mmol})$ in the dark. The reaction mixture was stirred at room temperature for $2 \mathrm{~h}$ and then filtered through a pad of Celite. The filtrate was diluted with EtOAc, washed with water, dried over $\mathrm{MgSO}_{4}$, filtered, and concentrated in vacuo. The resulting residue was purified by silica gel column 
chromatography (hexane/EtOAc, $8: 1)$ to give $12(1.37 \mathrm{~g}, 92 \%)$ as a waxy solid: $[\alpha]^{21}$ 89.8 (c 1.1, $\left.\mathrm{CHCl}_{3}\right) ;{ }^{1} \mathrm{H} \mathrm{NMR}\left(300 \mathrm{MHz}, \mathrm{CDCl}_{3}\right.$, mixture of rotamers) $\delta 1.45-1.53(\mathrm{~m}$, 15H), 2.09 (s, 3H), 3.99-4.20 (m, 3H), 5.81-5.83 (m, 1H); ${ }^{13} \mathrm{C}$ NMR (75 MHz, $\mathrm{CDCl}_{3}$, rotamer 1/rotamer 2) $\delta 20.8 / 20.9,23.1 / 24.4,25.9 / 26.7,28.2 / 28.3,47.2 / 47.6,59.5 / 59.7$, 63.4/63.5, 64.4/63.9, 75.5, 80.7, 95.0/94.3, 151.4/152.3, 169.5; HRMS (CI) calcd for $\mathrm{C}_{15} \mathrm{H}_{23} \mathrm{NO}_{5} \mathrm{Br}\left([\mathrm{M}+\mathrm{H}]^{+}\right)$376.0821, found 376.0762.

\section{(S)-tert-Butyl 4-[(R)-1-acetoxyhexadeca-2,4-diynyl]-2,2-dimethyloxazolidine-3-} carboxylate (14).

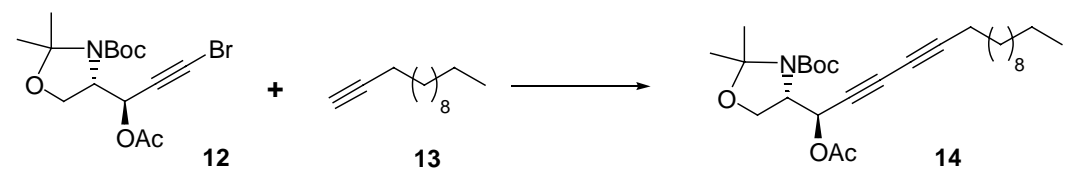

To a degassed solution of $12(200 \mathrm{mg}, 0.53 \mathrm{mmol})$, tridecyne $13(0.15 \mathrm{~mL}, 0.64$ $\mathrm{mmol}), \mathrm{Pd}\left(\mathrm{PPh}_{3}\right)_{2} \mathrm{Cl}_{2}(14 \mathrm{mg}, 0.02 \mathrm{mmol})$ and $\mathrm{CuI}(4 \mathrm{mg}, 0.02 \mathrm{mmol})$ in THF (5 mL) was added diisopropylamine $(0.20 \mathrm{~mL}, 1.41 \mathrm{mmol})$. The reaction mixture was stirred for $2 \mathrm{~h}$ at room temperature and then quenched with saturated $\mathrm{NH}_{4} \mathrm{Cl}$ solution. It was diluted with EtOAc, washed with brine, dried over $\mathrm{MgSO}_{4}$, filtered, and concentrated in vacuo. The resulting residue was purified by silica gel column chromatography (hexane/EtOAc, $15: 1)$ to give diyne $14(197 \mathrm{mg}, 78 \%)$ as a waxy solid: $[\alpha]^{25}-85.7(c$ $\left.0.2, \mathrm{CHCl}_{3}\right) ;{ }^{1} \mathrm{H} \mathrm{NMR}\left(300 \mathrm{MHz}, \mathrm{CDCl}_{3}\right.$, ca $6: 4$ mixture of rotamers) $\delta 0.82(\mathrm{t}, J=6.6$ $\mathrm{Hz}, 3 \mathrm{H}), 1.21-1.32(\mathrm{~m}, 18 \mathrm{H}), 1.40-1.48$ (m, 15H), 2.04 (s, 3H), 2.20-2.24 (m, 2H), 3.96$4.15(\mathrm{~m}, 3 \mathrm{H}), 5.78-5.82(\mathrm{~m}, 0.6 \mathrm{H}), 5.84-5.88(\mathrm{~m}, 0.4 \mathrm{H}) ;{ }^{13} \mathrm{C} \mathrm{NMR}\left(75 \mathrm{MHz}, \mathrm{CDCl}_{3}\right.$, rotamer 1/rotamer 2) $\delta 14.0,19.1,20.8,22.5,23.0 / 24.3,25.8 / 26.5,27.9,28.2,28.7,28.9$, $29.2,29.3,29.5,31.8,59.5 / 59.8,63.0,64.2 / 63.8,64.3 / 64.2,70.2,71.6 / 71.5,80.6$, 
82.4/82.0, 94.8/94.1, 151.3/152.1, 169.3; HRMS (CI) calcd for $\mathrm{C}_{28} \mathrm{H}_{46} \mathrm{NO}_{5}\left([\mathrm{M}+\mathrm{H}]^{+}\right)$ 476.3376, found 476.3382 .

(S)-tert-Butyl 4-[(R)-(E)-1-hydroxyhexadec-2-en-4-ynyl]-2,2-dimethyloxazolidine3-carboxylate (15).
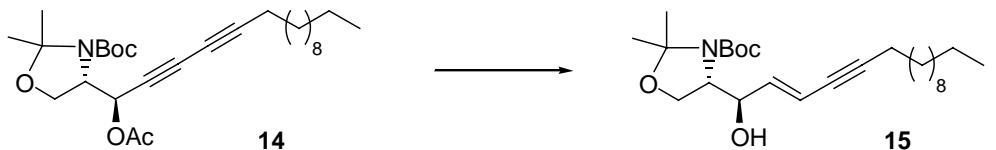

To a solution of diyne $14(100 \mathrm{mg}, 0.21 \mathrm{mmol})$ in THF (3 mL) was slowly added $\mathrm{LiAlH}_{4}\left(0.65 \mathrm{~mL}, 0.65 \mathrm{mmol}, 1.0 \mathrm{M}\right.$ solution in THF) at $0{ }^{\circ} \mathrm{C}$. The reaction mixture was slowly warmed to room temperature over $1 \mathrm{~h}$ and quenched with $10 \% \mathrm{NaOH}$. It was extracted with EtOAc three times. The combined organic layers were washed with brine, dried over $\mathrm{Na}_{2} \mathrm{SO}_{4}$, filtered, and concentrated in vacuo. The resulting residue was purified by silica gel column chromatography (hexane/EtOAc, 8:1) to give enyne 15 (87 $\mathrm{mg}, 95 \%)$ as a colorless oil: $[\alpha]^{25}-48.5\left(c\right.$ 1.3, $\left.\mathrm{CHCl}_{3}\right) ;{ }^{1} \mathrm{H} \mathrm{NMR}\left(300 \mathrm{MHz}, \mathrm{CDCl}_{3}\right.$, mixture of rotamers) $\delta 0.86(\mathrm{t}, J=6.6 \mathrm{~Hz}, 3 \mathrm{H}), 1.20-1.36(\mathrm{~m}, 18 \mathrm{H}), 1.41-1.54(\mathrm{~m}, 15 \mathrm{H})$, 2.23-2.28 (m, 2H), 3.81-4.37 (m, 5H), $5.78(\mathrm{br} \mathrm{d}, J=14.7 \mathrm{~Hz}, 1 \mathrm{H}), 6.01(\mathrm{dd}, \mathrm{J}=4.5$, $15.6 \mathrm{~Hz}, 1 \mathrm{H}) ;{ }^{13} \mathrm{C} \mathrm{NMR}\left(75 \mathrm{MHz}, \mathrm{CDCl}_{3}\right.$, rotamer 1/rotamer 2) $\delta 14.1,19.3,22.6,24.3$, $26.2,28.3,28.7,28.8,29.1,29.3,29.5,29.6,31.9,62.0 / 60.9,64.8 / 64.4,73.8 / 72.8,78.5$, 81.3, 91.2, 94.5, 111.7, 139.8/140.7, 154.3; HRMS (CI) calcd for $\mathrm{C}_{26} \mathrm{H}_{46} \mathrm{NO}_{4}\left([\mathrm{M}+\mathrm{H}]^{+}\right)$ 436.3427, found 436.3429 .

$(2 S, 3 R)-(E)-2-A m i n o o c t a d e c-4-e n-6-y n e-1,3-d i o l ~(2 a)$.

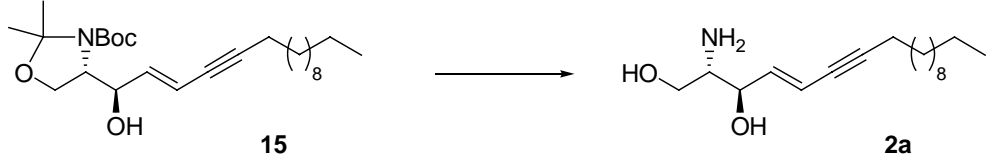


A solution of enyne $15(60 \mathrm{mg}, 0.13 \mathrm{mmol})$ in $\mathrm{CH}_{2} \mathrm{Cl}_{2}(2 \mathrm{~mL})$, TFA $(2 \mathrm{~mL})$, and $\mathrm{H}_{2} \mathrm{O}$ $(1 \mathrm{~mL})$ was stirred for $12 \mathrm{~h}$ at room temperature. The reaction mixture was quenched with saturated $\mathrm{NaHCO}_{3}$ solution and then extracted with EtOAc. The organic layer was washed with brine, dried over $\mathrm{MgSO}_{4}$, filtered and concentrated in vacuo. The residue was purified by silica gel column chromatography $\left(\mathrm{CH}_{2} \mathrm{Cl}_{2} / \mathrm{MeOH}, 10: 1\right.$, with $1 \%$ $\left.\mathrm{NH}_{4} \mathrm{OH}\right)$ to give $2 \mathrm{a}(36 \mathrm{mg}, 94 \%)$ as a white solid: $\mathrm{mp} 60.9-62.3{ }^{\circ} \mathrm{C} ;[\alpha]^{21}{ }_{\mathrm{D}}+16.5(c$ $\left.0.9, \mathrm{CH}_{3} \mathrm{OH}\right) ;{ }^{1} \mathrm{H}$ NMR $\left(300 \mathrm{MHz}, \mathrm{CD}_{3} \mathrm{OD}\right) \delta 0.89(\mathrm{t}, J=6.9 \mathrm{~Hz}, 3 \mathrm{H}), 1.29-1.42(\mathrm{~m}$, $16 \mathrm{H}), 1.45-1.55(\mathrm{~m}, 2 \mathrm{H}), 2.28(\mathrm{dt}, J=1.8,6.6 \mathrm{~Hz}, 2 \mathrm{H}), 2.80-2.86(\mathrm{~m} \mathrm{1H}), 3.50(\mathrm{dd}, J=$ $6.9,11.1 \mathrm{~Hz}, 1 \mathrm{H}), 3.65(\mathrm{dd}, J=4.5,11.1 \mathrm{~Hz}, 1 \mathrm{H}), 4.09(\mathrm{ddd}, J=1.2,6.6,6.6 \mathrm{~Hz}, 1 \mathrm{H})$, $5.75(\mathrm{dtd}, J=1.5,1.8,15.9 \mathrm{~Hz}, 1 \mathrm{H}), 6.04(\mathrm{dd}, J=6.6,15.9 \mathrm{~Hz}, 1 \mathrm{H}) ;{ }^{13} \mathrm{C} \mathrm{NMR}(75$ $\left.\mathrm{MHz}, \mathrm{CD}_{3} \mathrm{OD}\right) \delta 14.5,20.0,23.8,29.9,30.0,30.3,30.5,30.7,30.8$ (2C), 33.1, 58.0, 63.6, 73.9, 79.5, 92.0, 113.6, 141.7; IR $\left(\mathrm{CCl}_{4}\right) v_{\max } 3354,3256,2924,2851,2361,1574$, 1466, 1047, $960\left(\mathrm{~cm}^{-1}\right)$; HRMS (CI) calcd for $\mathrm{C}_{18} \mathrm{H}_{32} \mathrm{NO}_{2}\left([\mathrm{M}+\mathrm{H}]^{+}\right)$294.2433, found 294.2435.

\section{(2S,3R)-2-Aminooctadeca-4,6-diyne-1,3-diol (3a).}
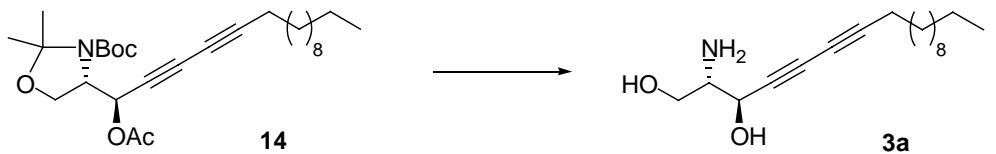

To a solution of diyne $14(60 \mathrm{mg}, 0.13 \mathrm{mmol})$ in anhydrous methanol $(3 \mathrm{~mL})$ was added sodium methoxide $(0.05 \mathrm{~mL}, 0.22 \mathrm{mmol}, 25 \mathrm{wt} \%$ solution in $\mathrm{MeOH})$. After being stirred at room temperature for $30 \mathrm{~min}$, the reaction mixture was diluted with EtOAc, washed with brine, dried over $\mathrm{MgSO}_{4}$, filtered, and concentrated in vacuo. A solution of the resulting crude residue in $\mathrm{CH}_{2} \mathrm{Cl}_{2}(1.4 \mathrm{~mL})$, TFA $(1.4 \mathrm{~mL})$, and $\mathrm{H}_{2} \mathrm{O}(0.7 \mathrm{~mL})$ was stirred for $12 \mathrm{~h}$ at room temperature. The reaction mixture was quenched with saturated 
$\mathrm{NaHCO}_{3}$ solution and then extracted with EtOAc. The organic layer was washed with brine, dried over $\mathrm{MgSO}_{4}$, filtered and concentrated in vacuo. The residue was purified by silica gel column chromatography $\left(\mathrm{CH}_{2} \mathrm{Cl}_{2} / \mathrm{MeOH}, 10: 1\right.$, with $\left.1 \% \mathrm{NH}_{4} \mathrm{OH}\right)$ to give 3a (34 mg, 92\%) as a white solid: $\mathrm{mp} 90.5-91.8{ }^{\circ} \mathrm{C} ;[\alpha]^{21}{ }_{\mathrm{D}}+3.0\left(c 0.6, \mathrm{CH}_{3} \mathrm{OH}\right) ;{ }^{1} \mathrm{H}$ NMR (300 MHz, CD 3 OD) $\delta 0.89(\mathrm{t}, J=6.9 \mathrm{~Hz}, 3 \mathrm{H}), 1.29-1.41(\mathrm{~m}, 16 \mathrm{H}), 1.47-1.56(\mathrm{~m}$, 2H), $2.29(\mathrm{t}, J=6.6 \mathrm{~Hz}, 2 \mathrm{H}), 2.85(\mathrm{ddd}, J=5.4,5.7,6.0 \mathrm{~Hz}, 1 \mathrm{H}), 3.56(\mathrm{dd}, J=6.0,11.1$ $\mathrm{Hz}, 1 \mathrm{H}), 3.64(\mathrm{dd}, J=5.7,11.1 \mathrm{~Hz}, 1 \mathrm{H}), 4.41(\mathrm{~d}, J=5.4 \mathrm{~Hz}, 1 \mathrm{H}) ;{ }^{13} \mathrm{C} \mathrm{NMR}(75 \mathrm{MHz}$, $\left.\mathrm{CD}_{3} \mathrm{OD}\right) \delta 14.5,19.8,23.8,29.4,29.9,30.2,30.5,30.7,30.8(2 \mathrm{C}), 33.1,58.5,63.6,65.0$, 65.4, 71.8, 75.4, 82.0; IR $\left(\mathrm{CCl}_{4}\right) v_{\max } 3342,3287,2920,2852,2357,2330,1026,939$ $\left(\mathrm{cm}^{-1}\right)$; HRMS (CI) calcd for $\mathrm{C}_{18} \mathrm{H}_{34} \mathrm{NO}_{2}\left([\mathrm{M}+\mathrm{H}]^{+}\right)$296.2589, found 296.2592.

\section{(S)-tert-Butyl 4-[(R)-1-acetoxy-5-bromopenta-2,4-diynyl]-2,2-}

\section{dimethyloxazolidine-3-carboxylate (16a).}

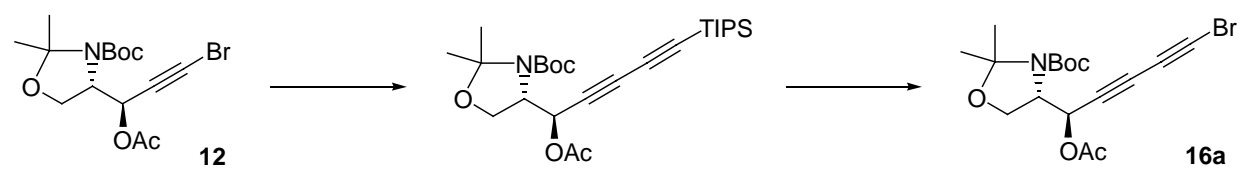

Following the same procedure as for 14, from bromo-acetylene $12(1.00 \mathrm{~g}, 2.66$ mmol) in THF (27 mL), (triisopropylsilyl)acetylene (0.7 mL, $3.2 \mathrm{mmol}), \mathrm{Pd}\left(\mathrm{PPh}_{3}\right)_{2} \mathrm{Cl}_{2}$ (56 mg, $0.08 \mathrm{mmol}), \mathrm{CuI}(15 \mathrm{mg}, 0.08 \mathrm{mmol})$, and diisopropylamine (0.8 $\mathrm{mL}, 5.7$ mmol), after a reaction time of $4 \mathrm{~h}$, TIPS-diyne $(1.14 \mathrm{~g}, 90 \%)$ was obtained as a brown oil: ${ }^{1} \mathrm{H}$ NMR $\left(300 \mathrm{MHz}, \mathrm{CDCl}_{3}\right.$, ca $6: 4$ mixture of rotamers) $\delta 1.06(\mathrm{~s}, 21 \mathrm{H}), 1.45-1.53$ (m, 15H), $2.10(\mathrm{~s}, 3 \mathrm{H}), 4.00-4.23(\mathrm{~m}, 3 \mathrm{H}), 5.80-5.84(\mathrm{~m}, 0.6 \mathrm{H}), 5.87-5.91(\mathrm{~m}, 0.4 \mathrm{H})$; HRMS (CI) calcd for $\mathrm{C}_{26} \mathrm{H}_{44} \mathrm{NO}_{5} \mathrm{Si}\left([\mathrm{M}+\mathrm{H}]^{+}\right)$478.2988, found 478.2983.

Following the same procedure as for 12, from the obtained TIPS-diyne (950 mg, 1.99 mmol) in acetonitrile (20 mL), NBS (425 mg, $2.39 \mathrm{mmol})$, and AgF (303 mg, 2.39 
$\mathrm{mmol})$, after a reaction time of $4 \mathrm{~h}$, bromo-diyne $\mathbf{1 6 a}(756 \mathrm{mg}, 95 \%)$ was obtained as a waxy solid: $[\alpha]^{21}{ }_{\mathrm{D}}-108.62\left(c 0.4, \mathrm{CHCl}_{3}\right) ;{ }^{1} \mathrm{H} \mathrm{NMR}\left(300 \mathrm{MHz}, \mathrm{CDCl}_{3}\right.$, ca 6:4 mixture of rotamers) $\delta 1.45-1.53(\mathrm{~m}, 15 \mathrm{H}), 2.10(\mathrm{~s}, 3 \mathrm{H}), 4.00-4.21(\mathrm{~m}, 3 \mathrm{H}), 5.79-83(\mathrm{~m}, 0.6 \mathrm{H})$, $5.86(\mathrm{~d}, J=3.3 \mathrm{~Hz}, 0.4 \mathrm{H}) ;{ }^{13} \mathrm{C} \mathrm{NMR}\left(75 \mathrm{MHz}, \mathrm{CDCl}_{3}\right.$, rotamer $1 /$ rotamer 2$) \delta 20.7$, 23.0/24.3, 25.9/26.6, 28.1, 42.4/42.9, 59.3/59.6, 62.8, 63.8/64.2, 64.4, 70.0, 71.2/71.4, 80.7, 94.2/94.9, 151.2/152.1, 169.2; $\mathrm{HRMS}$ (CI) calcd for $\mathrm{C}_{17} \mathrm{H}_{23} \mathrm{NO}_{5} \mathrm{Br}\left([\mathrm{M}+\mathrm{H}]^{+}\right)$ 400.0759, found 400.0758.

\section{(S)-tert-Butyl 4-[(R)-1-acetoxy-7-bromohepta-2,4,6-triynyl]-2,2-}

\section{dimethyloxazolidine-3-carboxylate (16b).}

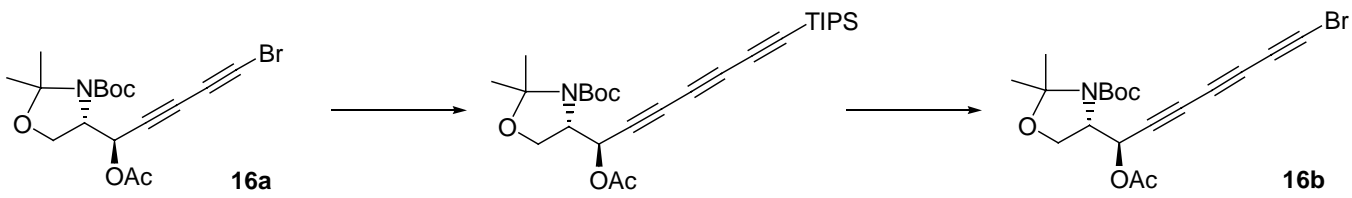

Following the same procedure as for 14, from bromo-diyne $16 \mathbf{a}(550 \mathrm{mg}, 1.37 \mathrm{mmol})$ in THF (14 mL), (triisopropylsilyl)acetylene (0.40 mL, $1.80 \mathrm{mmol}), \mathrm{Pd}\left(\mathrm{PPh}_{3}\right)_{2} \mathrm{Cl}_{2}(28$ mg, $0.04 \mathrm{mmol}), \mathrm{CuI}(8 \mathrm{mg}, 0.04 \mathrm{mmol})$, and diisopropylamine (0.4 mL, $2.83 \mathrm{mmol})$, after a reaction time of $5 \mathrm{~h}$, TIPS-triyne $(562 \mathrm{mg}, 82 \%)$ was obtained as a brown oil: ${ }^{1} \mathrm{H}$ NMR $\left(300 \mathrm{MHz}, \mathrm{CDCl}_{3}\right.$, ca 6:4 mixture of rotamers) $\delta 0.99(\mathrm{~s}, 21 \mathrm{H}), 1.37-1.43(\mathrm{~m}$, 15H), 2.00 and 2.01 (each s, total 3H), 3.91-4.13 (m, 3H), 5.76-5.80 (m, 0.6H), $5.82(\mathrm{~d}$, $J=3.0 \mathrm{~Hz}, 0.4 \mathrm{H})$; HRMS (CI) calcd for $\mathrm{C}_{28} \mathrm{H}_{44} \mathrm{NO}_{5} \mathrm{Si}\left([\mathrm{M}+\mathrm{H}]^{+}\right)$502.2988, found 502.2988 .

Following the same procedure as for 12, from the obtained TIPS-triyne (420 mg, 0.84 mmol) in acetonitrile (10 mL), NBS (179 mg, $1.01 \mathrm{mmol})$, and $\mathrm{AgF}$ (127 mg, 1.00 mmol), after a reaction time of $2 \mathrm{~h}$, bromo-triyne $\mathbf{1 6 b}(341 \mathrm{mg}, 96 \%)$ was obtained as a 
brown oil: $[\alpha]^{25}-72.4\left(c 0.2, \mathrm{CHCl}_{3}\right) ;{ }^{1} \mathrm{H}$ NMR (300 $\mathrm{MHz}, \mathrm{CDCl}_{3}$, ca 1:1 mixture of rotamers) $\delta 1.38-1.47(\mathrm{~m}, 15 \mathrm{H}), 2.02$ and 2.04 (each s, total $3 \mathrm{H}), 3.93-4.06(\mathrm{~m}, 2.5 \mathrm{H})$, 4.12-4.17 (m, 0.5H), 5.73-5.76 (m, 0.5H), $5.78(\mathrm{~d}, J=3.3 \mathrm{~Hz}, 0.5 \mathrm{H}) ;{ }^{13} \mathrm{C}$ NMR $(75$ $\mathrm{MHz}, \mathrm{CDCl}_{3}$, rotamer 1/rotamer 2) $\delta$ 20.6, 22.9/24.2, 25.9/26.6, 28.1, 42.5/42.1, $57.8 / 57.9,59.2 / 59.5,62.8 / 62.9,63.8 / 64.1,64.2 / 64.5,65.4 / 65.5,71.2 / 70.9,72.0 / 72.1$, 80.7/80.8, 94.8/94.1, 151.1/152.1, 169.0; HRMS (FAB) calcd for $\mathrm{C}_{19} \mathrm{H}_{23} \mathrm{NO}_{5} \mathrm{Br}$ $\left([\mathrm{M}+\mathrm{H}]^{+}\right) 424.0759$, found 424.0760 .

(S)-tert-Butyl 4-((R)-1-acetoxyhexadeca-2,4,6-triynyl)-2,2-dimethyloxazolidine-3carboxylate (18a).

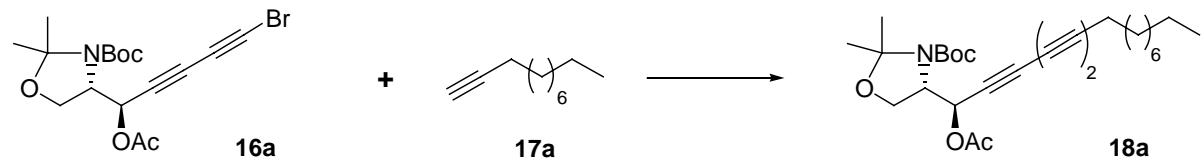

Following the same procedure as for $\mathbf{1 4}$, from bromo-diyne $\mathbf{1 6 a}(180 \mathrm{mg}, 0.45 \mathrm{mmol})$ in THF (5 mL), undecyne 17a $(0.10 \mathrm{~mL}, 0.51 \mathrm{mmol}), \mathrm{Pd}\left(\mathrm{PPh}_{3}\right)_{2} \mathrm{Cl}_{2}(7 \mathrm{mg}, 0.01 \mathrm{mmol})$, $\mathrm{CuI}(2 \mathrm{mg}, 0.01 \mathrm{mmol})$, and diisopropylamine $(0.10 \mathrm{~mL}, 0.70 \mathrm{mmol})$, after a reaction time of $5 \mathrm{~h}$, triyne 18a (144 mg, 68\%) was obtained as a waxy solid: $[\alpha]^{25}-93.0(c 0.9$, $\left.\mathrm{CHCl}_{3}\right) ;{ }^{1} \mathrm{H} \mathrm{NMR}\left(300 \mathrm{MHz}, \mathrm{CDCl}_{3}\right.$, ca $6: 4$ mixture of rotamers) $\delta 0.84(\mathrm{t}, J=6.6 \mathrm{~Hz}$, 3H), 1.22-1.33 (m, 14H), 1.41-1.49 (m, 15H), 2.05 and 2.06 (each s, total 3H), $2.25(\mathrm{t}, J$ $=6.6 \mathrm{~Hz}, 2 \mathrm{H}), 3.97-4.18(\mathrm{~m}, 3 \mathrm{H}), 5.80(\mathrm{~d}, J=1.8 \mathrm{~Hz}, 0.6 \mathrm{H}), 5.86(\mathrm{~d}, J=3.0 \mathrm{~Hz}, 0.4 \mathrm{H})$; ${ }^{13} \mathrm{C}$ NMR (75 MHz, $\mathrm{CDCl}_{3}$, rotamer 1/rotamer 2) $\delta 14.0,19.3,20.7,22.5,23.0 / 24.3$, 25.9/26.6, 27.8, 28.2, 28.7, 28.9, 29.1, 29.3, 31.7, 58.4/58.5, 59.4/59.7, 63.0, 63.8/64.6, 64.3/64.9, 65.1/65.2, 71.5/71.8, 71.6, 80.7, 81.9/81.6, 94.9/94.2, 151.2/152.2, 169.2; HRMS (FAB) calcd for $\mathrm{C}_{28} \mathrm{H}_{42} \mathrm{NO}_{5}\left([\mathrm{M}+\mathrm{H}]^{+}\right)$472.3063, found 472.3062. 


\section{(S)-tert-Butyl 4-((R)-1-acetoxyhexadeca-2,4,6,8-tetraynyl)-2,2-}

\section{dimethyloxazolidine-3-carboxylate (18b).}

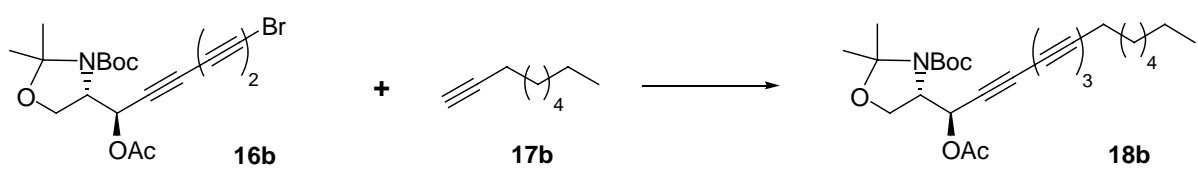

Following the same procedure as for $\mathbf{1 4}$, from bromo-triyne $\mathbf{1 6 b}(320 \mathrm{mg}, 0.75 \mathrm{mmol})$ in THF (8 mL), nonyne 17b $(0.15 \mathrm{~mL}, 0.91 \mathrm{mmol}), \mathrm{Pd}\left(\mathrm{PPh}_{3}\right)_{2} \mathrm{Cl}_{2}(14 \mathrm{mg}, 0.02 \mathrm{mmol})$, $\mathrm{CuI}(4 \mathrm{mg}, 0.02 \mathrm{mmol})$, and diisopropylamine $(0.22 \mathrm{~mL}, 1.6 \mathrm{mmol})$, after a reaction time of $3 \mathrm{~h}$, tetrayne $\mathbf{1 8 b}(221 \mathrm{mg}, 63 \%)$ was obtained as a waxy solid: $[\alpha]^{25}{ }_{\mathrm{D}}-90.7(c$ 1.5, $\left.\mathrm{CHCl}_{3}\right) ;{ }^{1} \mathrm{H} \mathrm{NMR}\left(300 \mathrm{MHz}, \mathrm{CDCl}_{3}\right.$, ca $6: 4$ mixture of rotamers) $\delta 0.86(\mathrm{t}, J=6.9$ $\mathrm{Hz}, 3 \mathrm{H}), 1.24-1.37(\mathrm{~m}, 10 \mathrm{H}), 1.43-1.51(\mathrm{~m}, 15 \mathrm{H}), 2.08$ and 2.09 (each s, total $3 \mathrm{H}), 2.29$ $(\mathrm{t}, J=6.9 \mathrm{~Hz}, 2 \mathrm{H}), 3.99-4.21(\mathrm{~m}, 3 \mathrm{H}), 5.79-5.82(\mathrm{~m}, 0.6 \mathrm{H}), 5.85(\mathrm{~d}, J=3.6 \mathrm{~Hz}, 0.4 \mathrm{H})$; ${ }^{13} \mathrm{C}$ NMR (75 MHz, $\mathrm{CDCl}_{3}$, rotamer 1/rotamer 2) $\delta 14.0,19.4,20.7,22.5,23.0 / 24.3$, 26.0/26.7, 27.8, 28.2, 28.6, 28.7, 31.5, 59.4/59.3, 59.5/59.8, 59.6, 63.1, 63.2/63.4, 63.7/63.9, 64.4/64.7, 65.4/65.1, 71.6/71.4, 72.4/72.5, 81.0, 82.1/81.8, 95.0/94.3, 151.3/152.2, 169.2; HRMS (FAB) calcd for $\mathrm{C}_{28} \mathrm{H}_{38} \mathrm{NO}_{5}\left([\mathrm{M}+\mathrm{H}]^{+}\right)$468.2750, found 468.2761.

\section{General procedure for $3 b$ and $3 c$.}
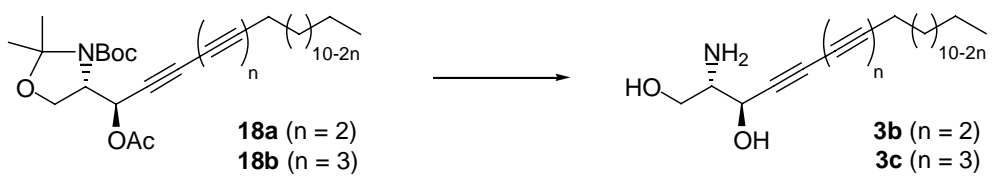

To a solution of polyyne $\mathbf{1 8}$ in anhydrous methanol $(0.05 \mathrm{M})$ was added sodium methoxide (1.5 equiv, $25 \mathrm{wt} \%$ solution in $\mathrm{MeOH})$. After being stirred at room temperature for $1 \mathrm{~h}$, the reaction mixture was diluted with EtOAc, washed with brine, dried over $\mathrm{MgSO}_{4}$, filtered, and concentrated in vacuo. A solution of the resulting crude 
residue in $\mathrm{CH}_{2} \mathrm{Cl}_{2} / \mathrm{TFA} / \mathrm{H}_{2} \mathrm{O}(2 / 2 / 1,0.1 \mathrm{M})$ was stirred for $10-12 \mathrm{~h}$ at room temperature. The reaction mixture was quenched with saturated $\mathrm{NaHCO}_{3}$ solution and then extracted with EtOAc. The organic layer was washed with brine, dried over $\mathrm{MgSO}_{4}$, filtered and concentrated in vacuo. The residue was purified by silica gel column chromatography $\left(\mathrm{CH}_{2} \mathrm{Cl}_{2} / \mathrm{MeOH}, 10: 1\right.$, with $\left.1 \% \mathrm{NH}_{4} \mathrm{OH}\right)$ to 3 .

(2S,3R)-2-Aminooctadeca-4,6,8-triyne-1,3-diol (3b, $\mathbf{n}=\mathbf{2}) .91 \%$ yield (39 $\mathrm{mg})$ as a light yellow solid: mp $110.8-114.7{ }^{\circ} \mathrm{C} ;[\alpha]^{25}{ }_{\mathrm{D}}+4.5\left(c 0.9, \mathrm{CH}_{3} \mathrm{OH}\right) ;{ }^{1} \mathrm{H}$ NMR $(300$ $\left.\mathrm{MHz}, \mathrm{CD}_{3} \mathrm{OD}\right) \delta 0.90(\mathrm{t}, J=6.9 \mathrm{~Hz}, 3 \mathrm{H}), 1.30-1.42(\mathrm{~m}, 12 \mathrm{H}), 1.46-1.58(\mathrm{~m}, 2 \mathrm{H}), 2.33$ $(\mathrm{t}, J=6.9 \mathrm{~Hz}, 2 \mathrm{H}), 2.87-2.89(\mathrm{~m}, 1 \mathrm{H}), 3.60(\mathrm{dd}, J=5.7,11.1 \mathrm{~Hz}, 1 \mathrm{H}), 3.64(\mathrm{dd}, J=5.4$, $11.1 \mathrm{~Hz}, 1 \mathrm{H}), 4.44(\mathrm{~d}, J=5.4 \mathrm{~Hz}, 1 \mathrm{H}) ;{ }^{13} \mathrm{C} \mathrm{NMR}\left(75 \mathrm{MHz}, \mathrm{CD}_{3} \mathrm{OD}\right) \delta 14.5,19.8,23.7$, $29.1,29.9,30.1,30.4,30.6,33.0,58.5,59.7,63.6,64.4,65.1,65.9,71.4,77.4,82.3$; IR $\left(\mathrm{CCl}_{4}\right) v_{\max } 3354,3238,2918,2849,2357,2204,1028,952\left(\mathrm{~cm}^{-1}\right)$; HRMS (FAB) calcd for $\mathrm{C}_{18} \mathrm{H}_{28} \mathrm{NO}_{2}\left([\mathrm{M}+\mathrm{H}]^{+}\right)$290.2120, found 290.2124.

(2S,3R)-2-Aminooctadeca-4,6,8,10-tetrayne-1,3-diol $(3 \mathbf{c}, \mathbf{n}=\mathbf{3}) .85 \%$ yield (12 mg) as a light yellow solid: mp 108.6-110.4 ${ }^{\circ} \mathrm{C} ;[\alpha]^{25}{ }_{\mathrm{D}}+1.9\left(c 0.2, \mathrm{CH}_{3} \mathrm{OH}\right) ;{ }^{1} \mathrm{H}$ NMR $(300$ $\left.\mathrm{MHz}, \mathrm{CD}_{3} \mathrm{OD}\right) \delta 0.91(\mathrm{t}, J=6.9 \mathrm{~Hz}, 3 \mathrm{H}), 1.29-1.41(\mathrm{~m}, 8 \mathrm{H}), 1.49-1.59(\mathrm{~m}, 2 \mathrm{H}), 2.36(\mathrm{t}$, $J=6.9 \mathrm{~Hz}, 2 \mathrm{H}), 2.91($ app. q, $J=5.7 \mathrm{~Hz}, 1 \mathrm{H}), 3.58(\mathrm{dd}, J=6.3,10.8 \mathrm{~Hz}, 1 \mathrm{H}), 3.64(\mathrm{dd}$, $J=5.7,10.8 \mathrm{~Hz}, 1 \mathrm{H}), 4.47(\mathrm{~d}, J=6.0 \mathrm{~Hz}, 1 \mathrm{H}) ;{ }^{13} \mathrm{C} \mathrm{NMR}\left(75 \mathrm{MHz}, \mathrm{CD}_{3} \mathrm{OD}\right) \delta 14.4$, 19.9, 23.7, 29.0, 29.8, 29.9, 32.8, 58.5, 60.2, 60.8, 63.4, 63.8, 64.2, 65.0, 65.8, 71.0, 78.7, 83.1; IR $\left(\mathrm{CCl}_{4}\right) v_{\max } 3342,3244,2912,2862,2361,2204,1026,953\left(\mathrm{~cm}^{-1}\right)$; HRMS (FAB) calcd for $\mathrm{C}_{18} \mathrm{H}_{24} \mathrm{NO}_{2}\left([\mathrm{M}+\mathrm{H}]^{+}\right)$286.1807, found 286.1799.

\section{General procedure for $2 b$ and $2 c$.}




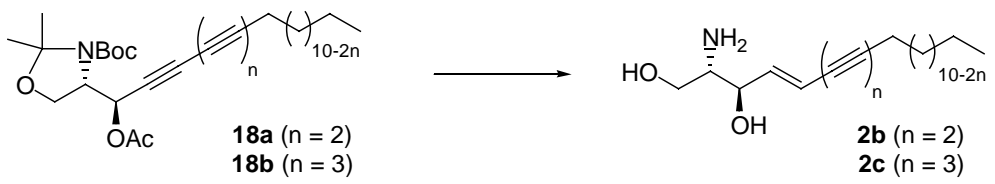

To a solution of 18 in $\operatorname{THF}(0.05 \mathrm{M})$ was added $\mathrm{LiAlH}_{4}$ (3 equiv) at $0{ }^{\circ} \mathrm{C}$. The reaction was then slowly warmed to room temperature over 1-2 $\mathrm{h}$ and quenched with $10 \% \mathrm{NaOH}$. The reaction mixture was extracted with EtOAc three times. The combined organic layers were washed with brine, dried over $\mathrm{Na}_{2} \mathrm{SO}_{4}$, filtered, and concentrated in vacuo. A solution of the resulting crude residue in $\mathrm{CH}_{2} \mathrm{Cl}_{2} / \mathrm{TFA} / \mathrm{H}_{2} \mathrm{O}(2 / 2 / 1,0.1 \mathrm{M})$ was stirred for 10-12 $\mathrm{h}$ at room temperature. The reaction mixture was quenched with saturated $\mathrm{NaHCO}_{3}$ solution and then extracted with EtOAc. The organic layer was washed with brine, dried over $\mathrm{MgSO}_{4}$, filtered and concentrated in vacuo. The residue was purified by silica gel column chromatography $\left(\mathrm{CH}_{2} \mathrm{Cl}_{2} / \mathrm{MeOH}, 10: 1\right.$, with $1 \%$ $\mathrm{NH}_{4} \mathrm{OH}$ ) to give 2 .

(2S,3R)-(E)-2-Aminooctadeca-4-en-6,8-diyne-1,3-diol $(\mathbf{2 b}, \mathbf{n}=\mathbf{2}) .94 \%$ yield (32 mg) as a light yellow solid: mp 87.1-90.3 ${ }^{\circ} \mathrm{C} ;[\alpha]^{25}-0.59\left(c 0.2, \mathrm{CH}_{3} \mathrm{OH}\right) ;{ }^{1} \mathrm{H}$ NMR $\left(300 \mathrm{MHz}, \mathrm{CD}_{3} \mathrm{OD}\right) \delta 0.90(\mathrm{t}, J=6.6 \mathrm{~Hz}, 3 \mathrm{H}), 1.30-1.39(\mathrm{~m}, 12 \mathrm{H}), 1.47-1.57(\mathrm{~m}, 2 \mathrm{H})$, $2.32(\mathrm{t}, J=6.9 \mathrm{~Hz}, 2 \mathrm{H}), 2.86-2.92(\mathrm{~m}, 1 \mathrm{H}), 3.51(\mathrm{dd}, J=6.9,11.1 \mathrm{~Hz}, 1 \mathrm{H}), 3.64(\mathrm{dd}, J$ $=4.5,11.1 \mathrm{~Hz}, 1 \mathrm{H}), 4.16-4.20(\mathrm{~m}, 1 \mathrm{H}), 5.84(\mathrm{~d}, J=15.9 \mathrm{~Hz}, 1 \mathrm{H}), 6.32(\mathrm{dd}, J=5.7$, $15.9 \mathrm{~Hz}, 1 \mathrm{H}) ;{ }^{13} \mathrm{C}$ NMR (75 MHz, $\left.\mathrm{CD}_{3} \mathrm{OD}\right) \delta 14.5,20.0,23.8,29.4,29.9,30.1,30.4$, 30.6, 33.0, 58.0, 63.0, 65.9, 73.2, 73.7, 76.1, 85.2, 111.6, 146.5; IR $\left(\mathrm{CCl}_{4}\right) v_{\max } 3354$, 3294, 2924, 2851, 2363, 2229, 1579, 1464, 1049, $963\left(\mathrm{~cm}^{-1}\right)$; HRMS (FAB) calcd for $\mathrm{C}_{18} \mathrm{H}_{30} \mathrm{NO}_{2}\left([\mathrm{M}+\mathrm{H}]^{+}\right)$292.2277, found 292.2268.

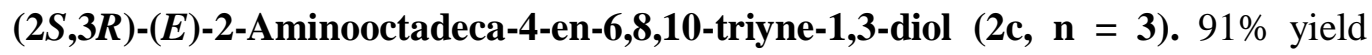
(21 mg) as a light yellow solid: mp 88.9-90.5 ${ }^{\circ} \mathrm{C} ;[\alpha]^{25}{ }_{\mathrm{D}}-0.56\left(c 0.5, \mathrm{CH}_{3} \mathrm{OH}\right) ;{ }^{1} \mathrm{H} \mathrm{NMR}$ $\left(300 \mathrm{MHz}, \mathrm{CD}_{3} \mathrm{OD}\right) \delta 0.90(\mathrm{t}, J=6.6 \mathrm{~Hz}, 3 \mathrm{H}), 1.29-1.42(\mathrm{~m}, 8 \mathrm{H}), 1.49-1.59(\mathrm{~m}, 2 \mathrm{H})$, 
$2.35(\mathrm{t}, J=6.9 \mathrm{~Hz}, 2 \mathrm{H}), 2.80-2.86(\mathrm{~m} 1 \mathrm{H}), 3.49(\mathrm{dd}, J=6.6,10.8 \mathrm{~Hz}, 1 \mathrm{H}), 3.62(\mathrm{dd}, J=$ 5.1, $11.4 \mathrm{~Hz}, 1 \mathrm{H}), 4.15-4.19(\mathrm{~m}, 1 \mathrm{H}), 5.87(\mathrm{~d}, J=15.0 \mathrm{~Hz}, 1 \mathrm{H}), 6.49(\mathrm{dd}, J=5.7,16.2$ $\mathrm{Hz}, 1 \mathrm{H}) ;{ }^{13} \mathrm{C}$ NMR $\left(75 \mathrm{MHz}, \mathrm{CD}_{3} \mathrm{OD}\right) \delta 14.4,20.0,23.7,29.2,29.8,29.9,32.9,58.0$, $60.0,63.8,66.1,67.6,73.7,75.0,75.5,83.7,110.2,149.8 ; \mathrm{IR}\left(\mathrm{CCl}_{4}\right) v_{\max } 3348,3260$, 2934, 2851, 2382, 2216, 1578, 1462, 1047, $958\left(\mathrm{~cm}^{-1}\right)$; HRMS (FAB) calcd for $\mathrm{C}_{18} \mathrm{H}_{26} \mathrm{NO}_{2}\left([\mathrm{M}+\mathrm{H}]^{+}\right)$288.1964, found 288.1966. 


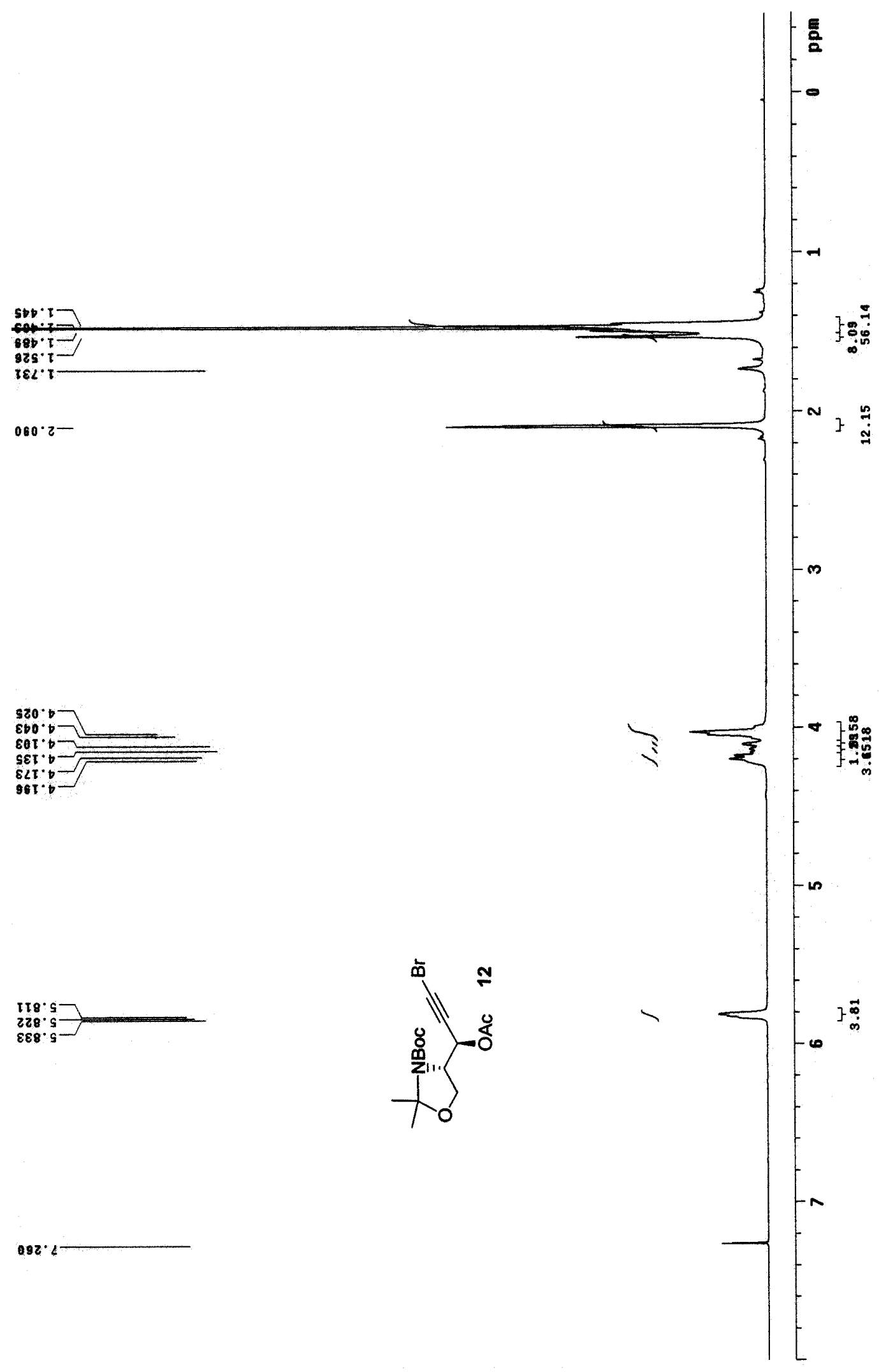



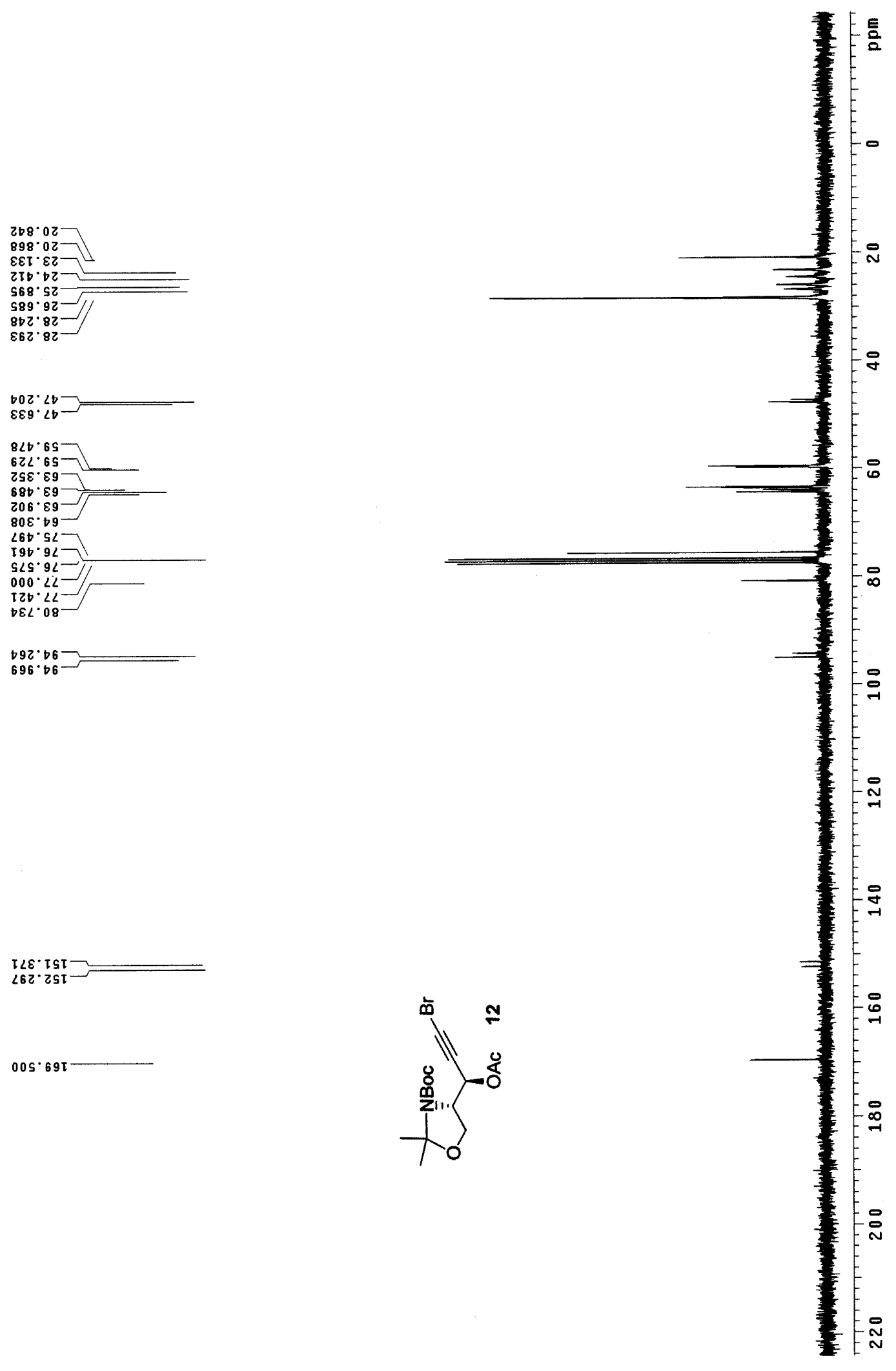

$00 S \cdot 69 I \ldots \ldots$ 


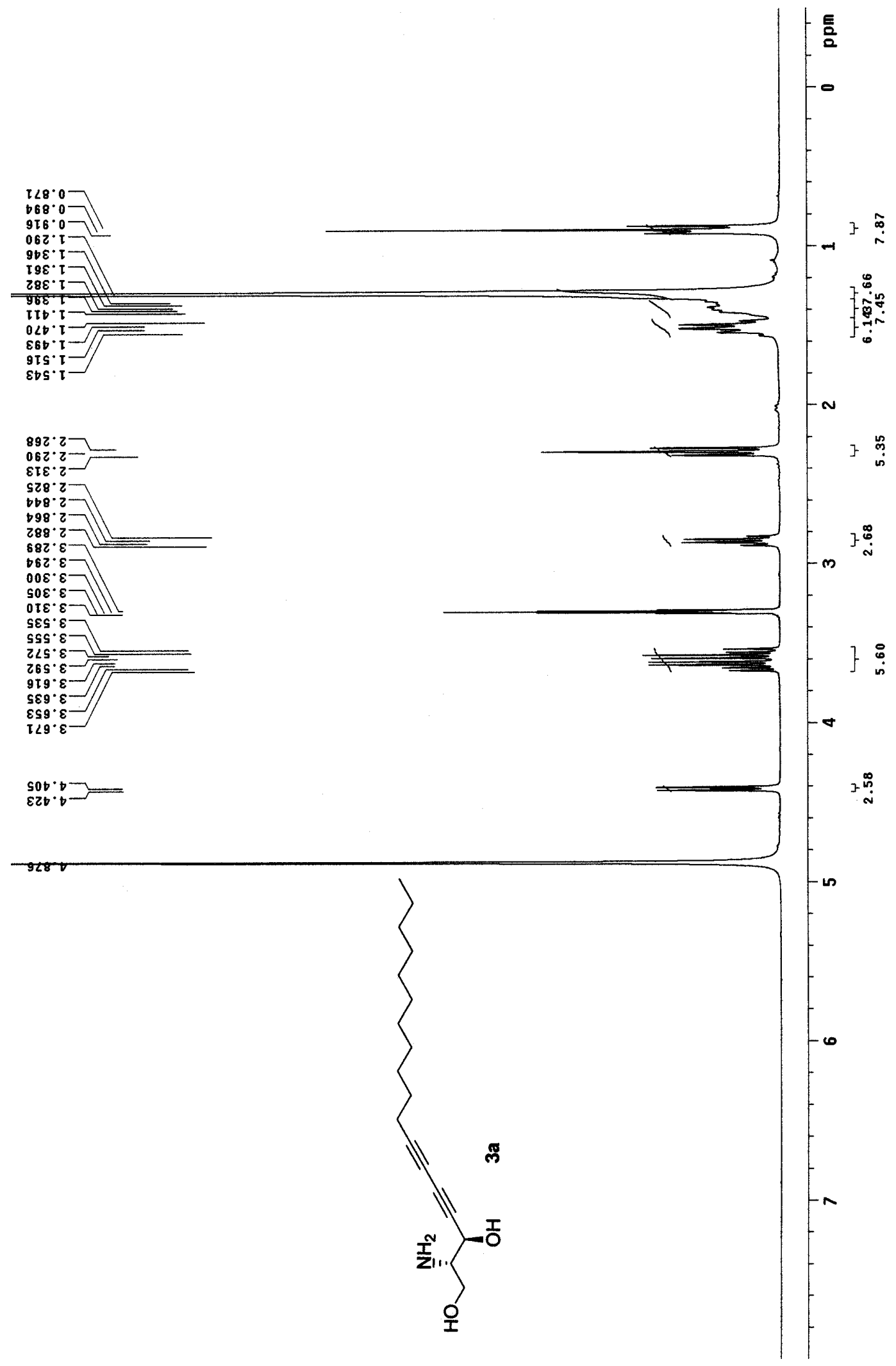



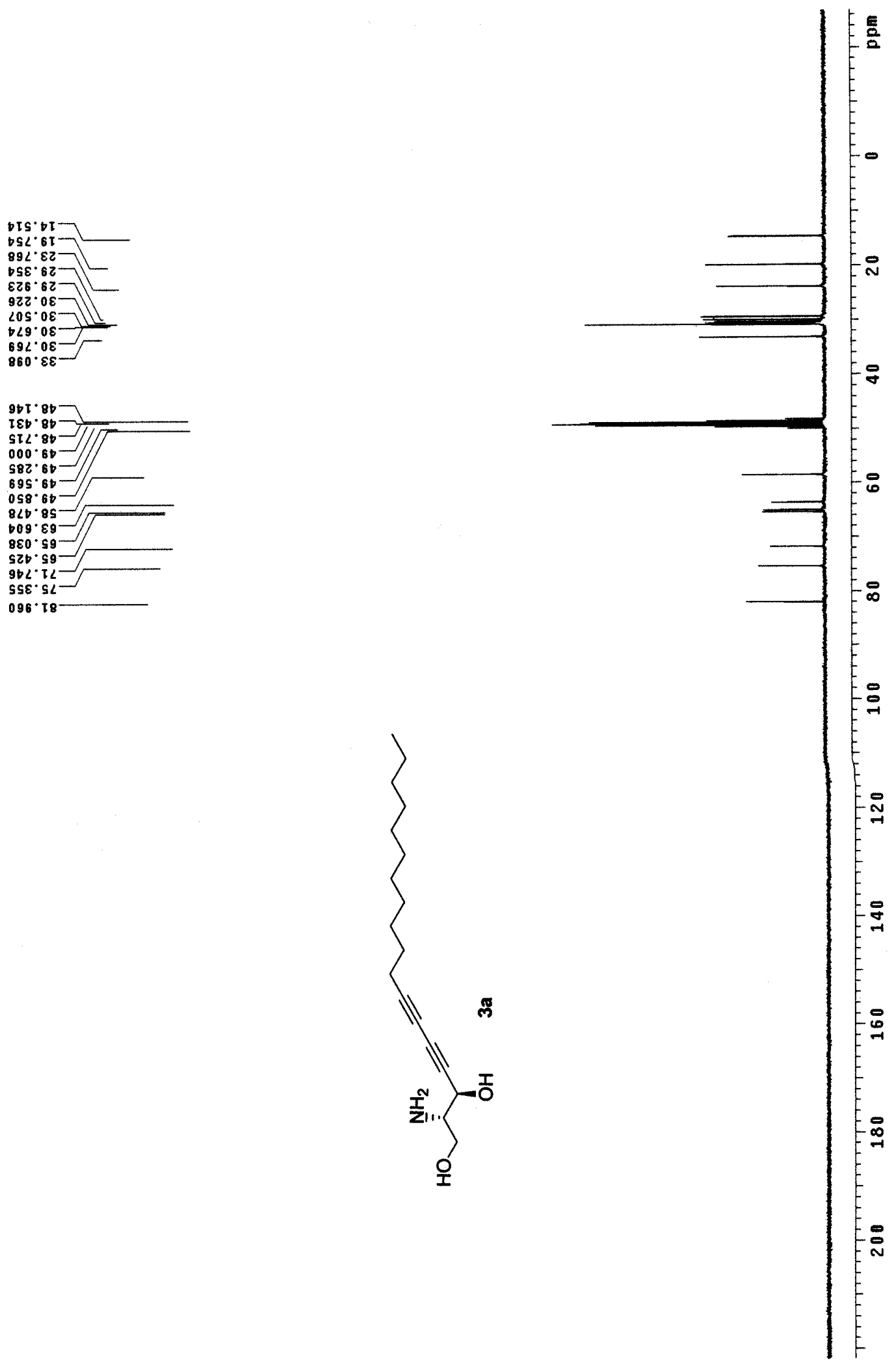


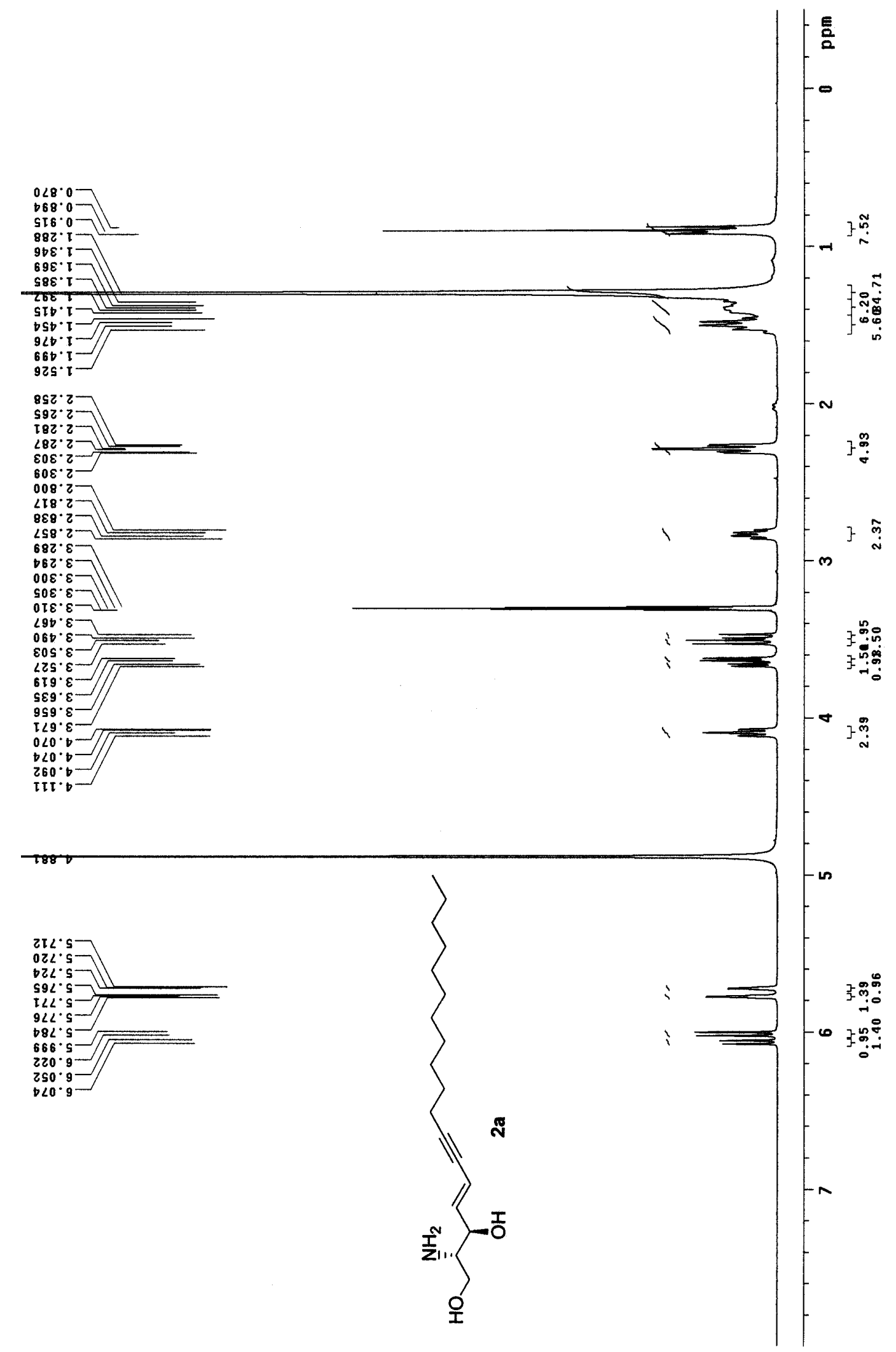




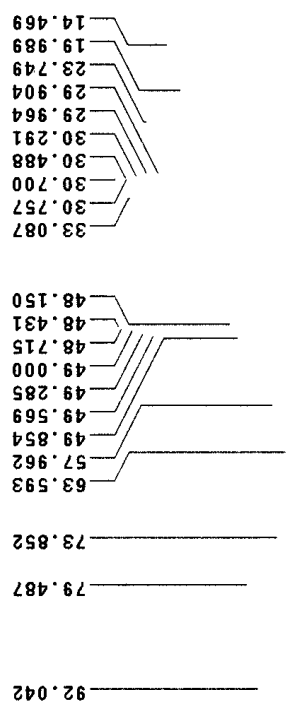

$\angle 6 S^{\circ} \varepsilon I I$

$6 t L^{\circ} โ b t \cdots$

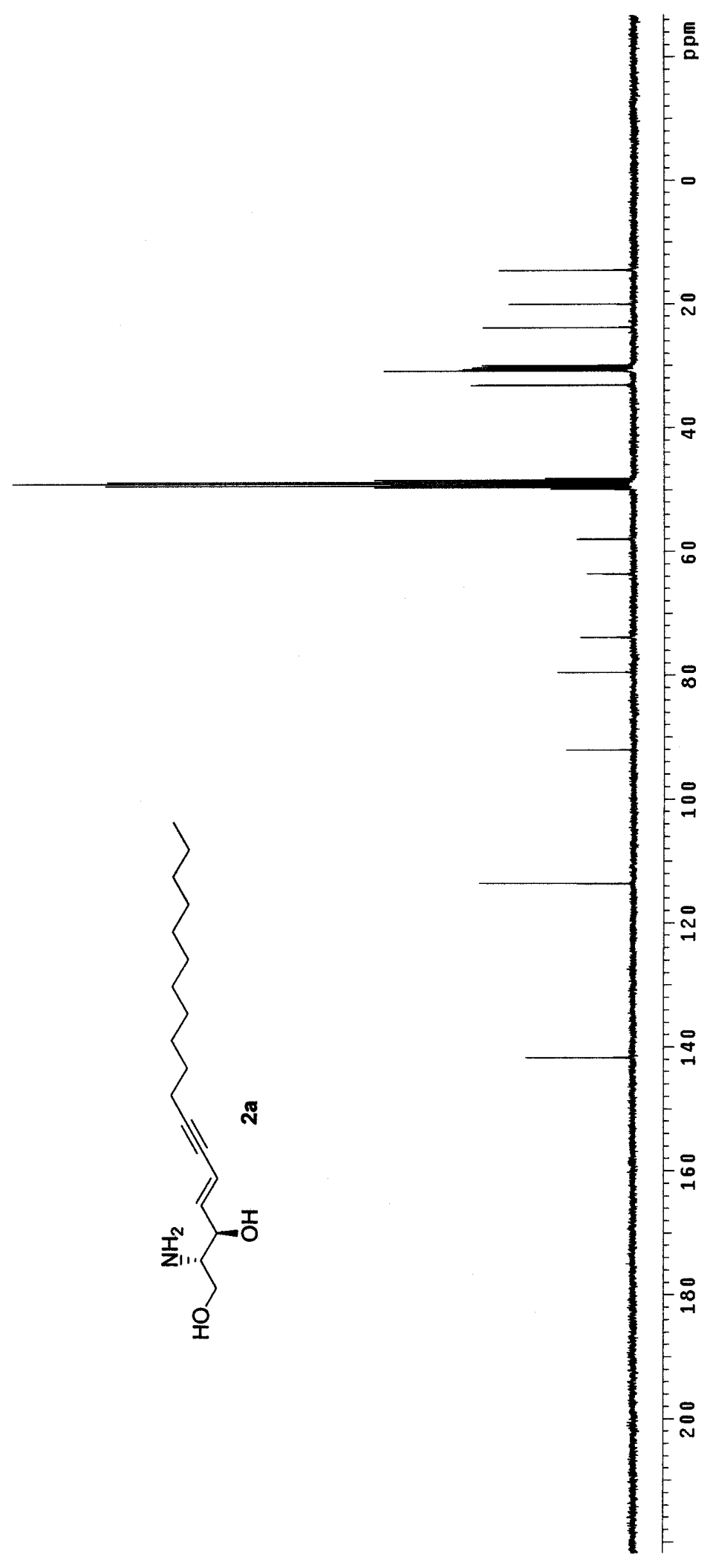




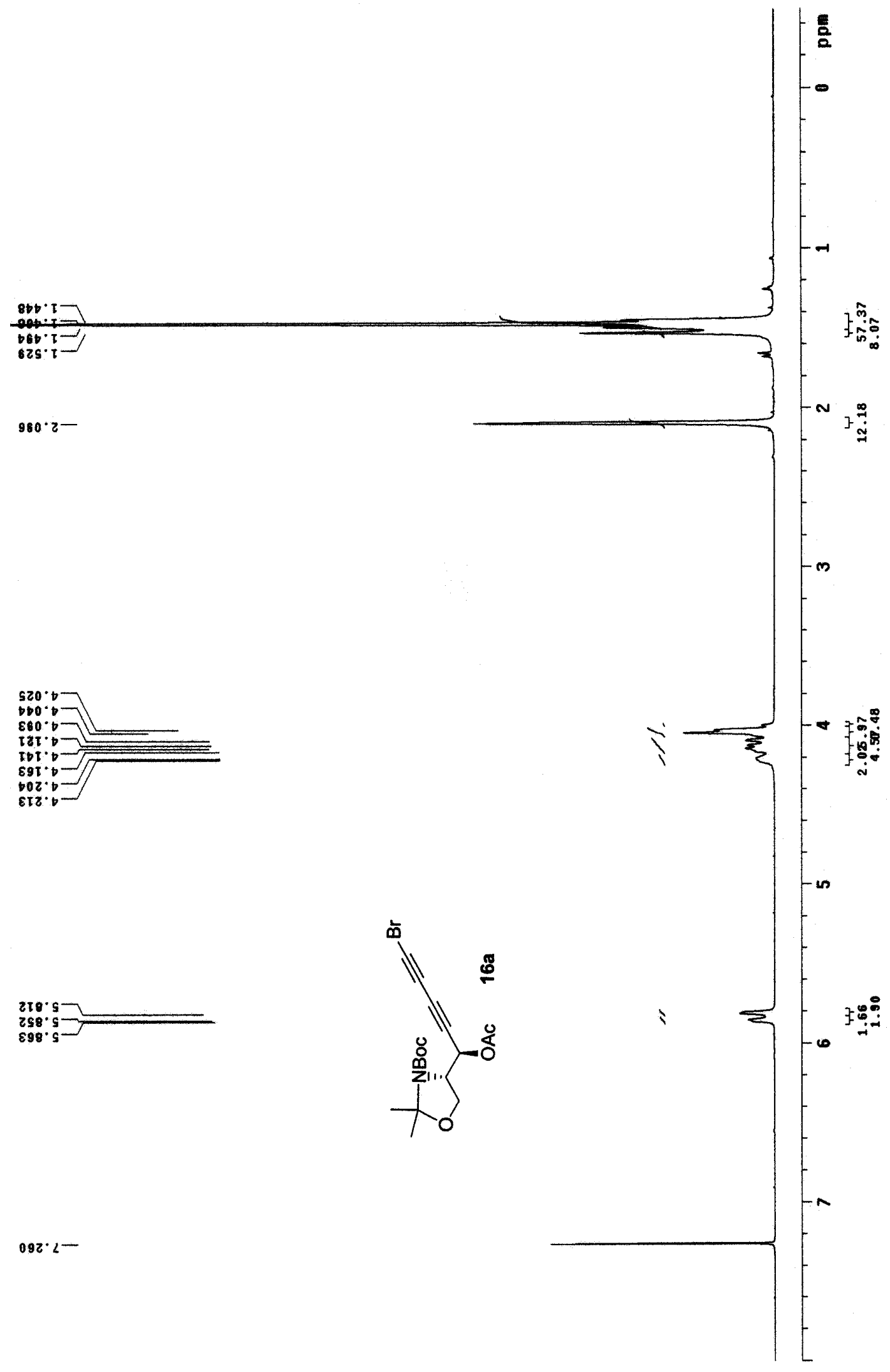




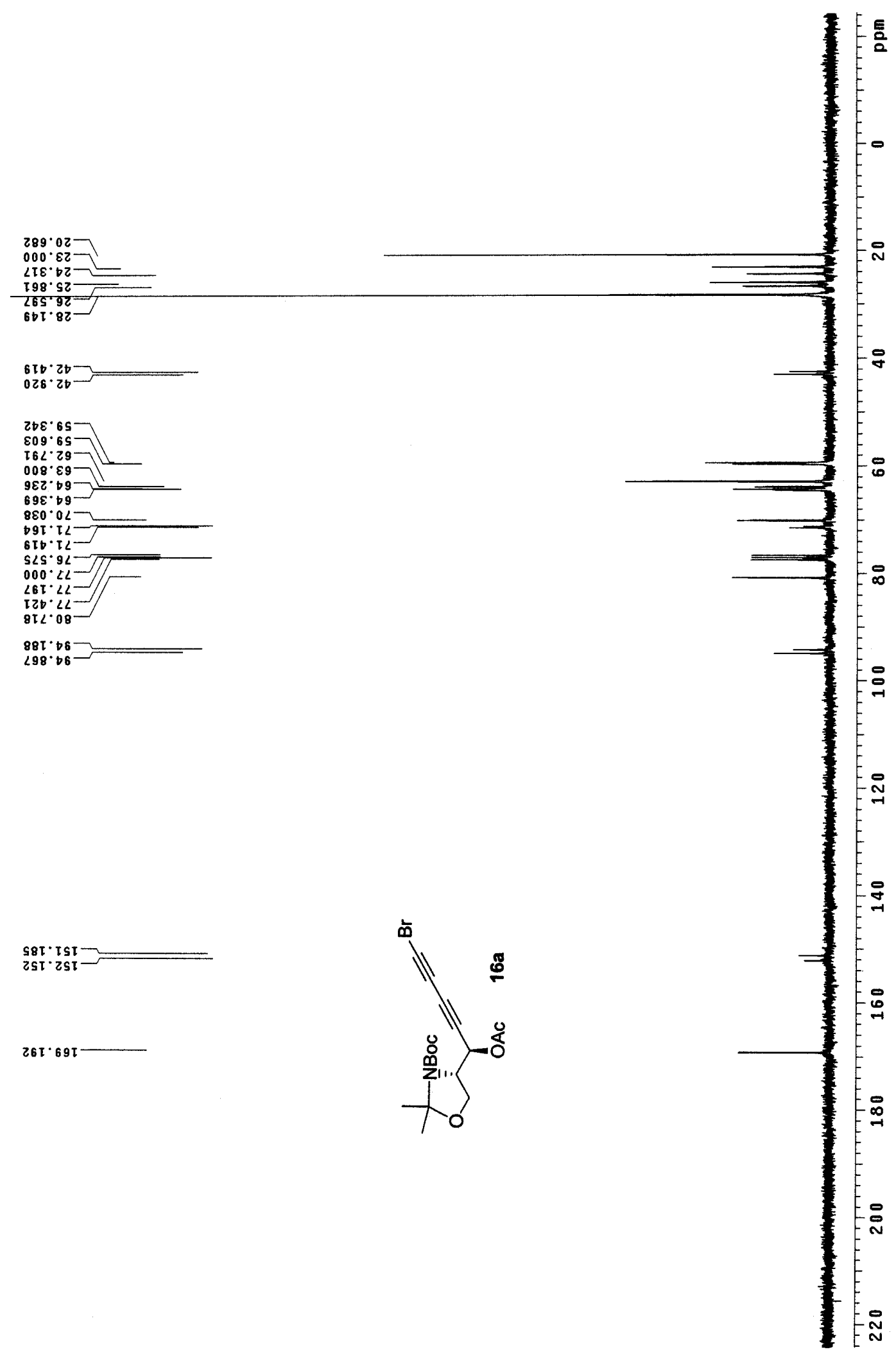




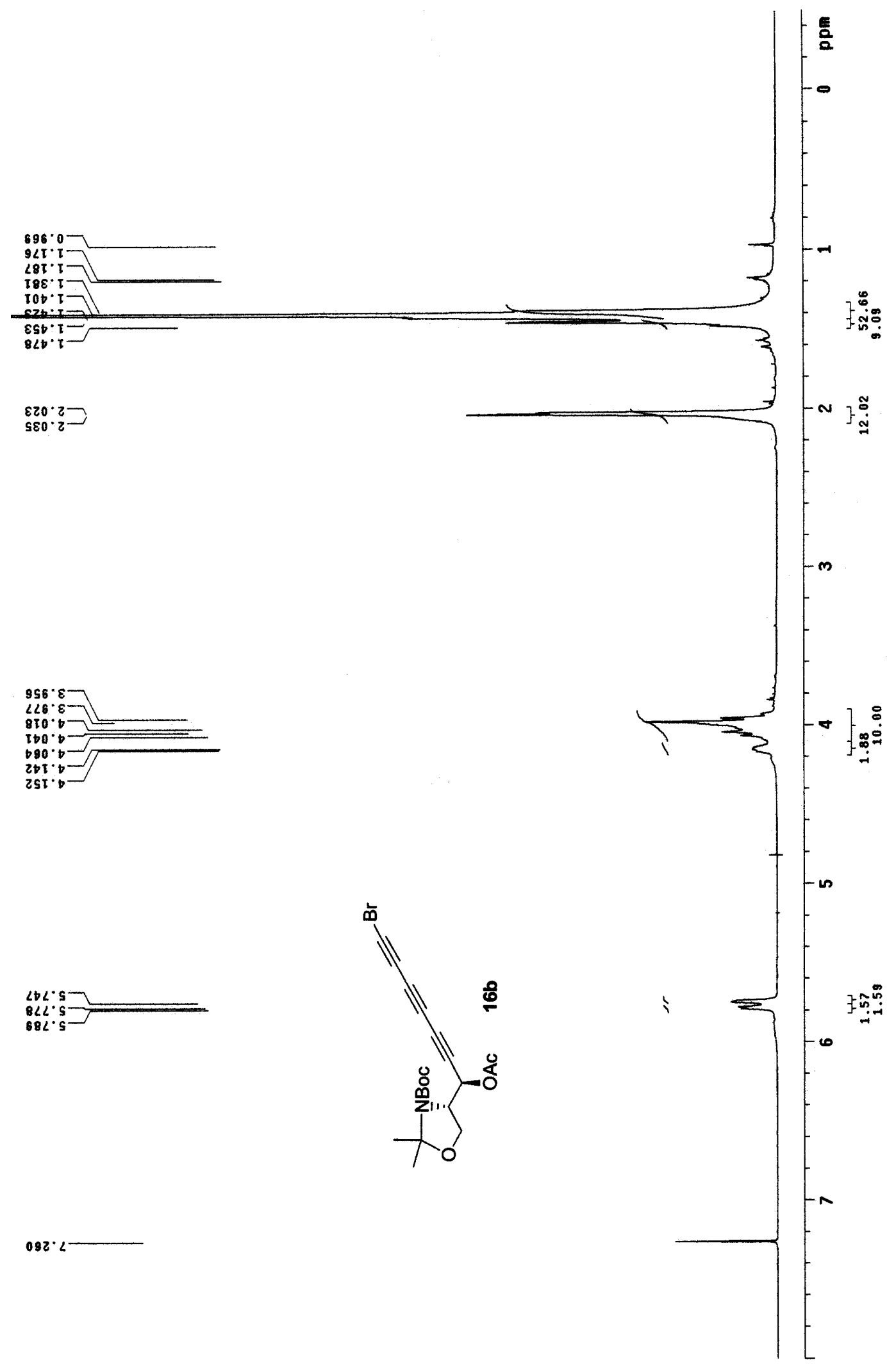




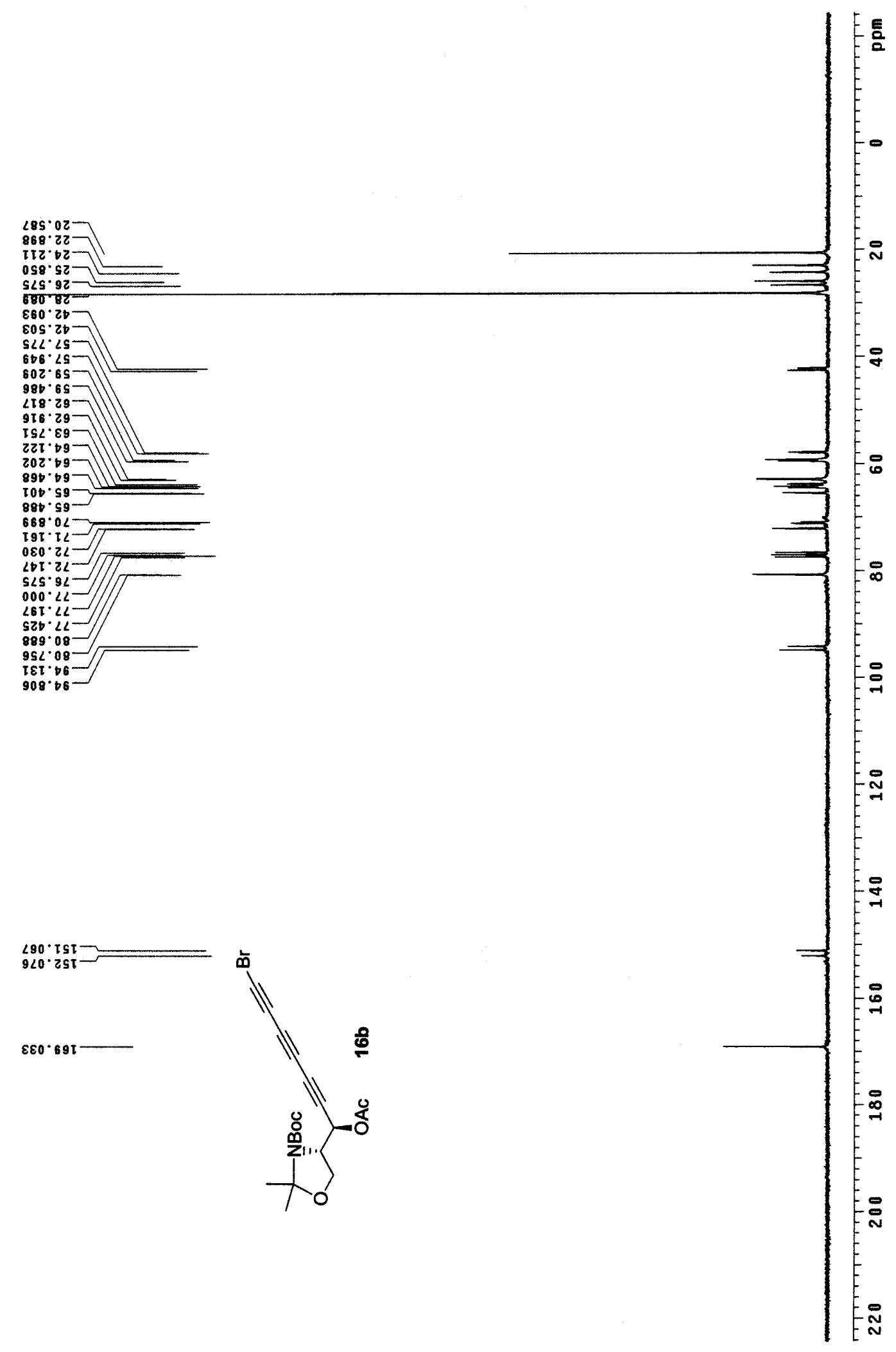




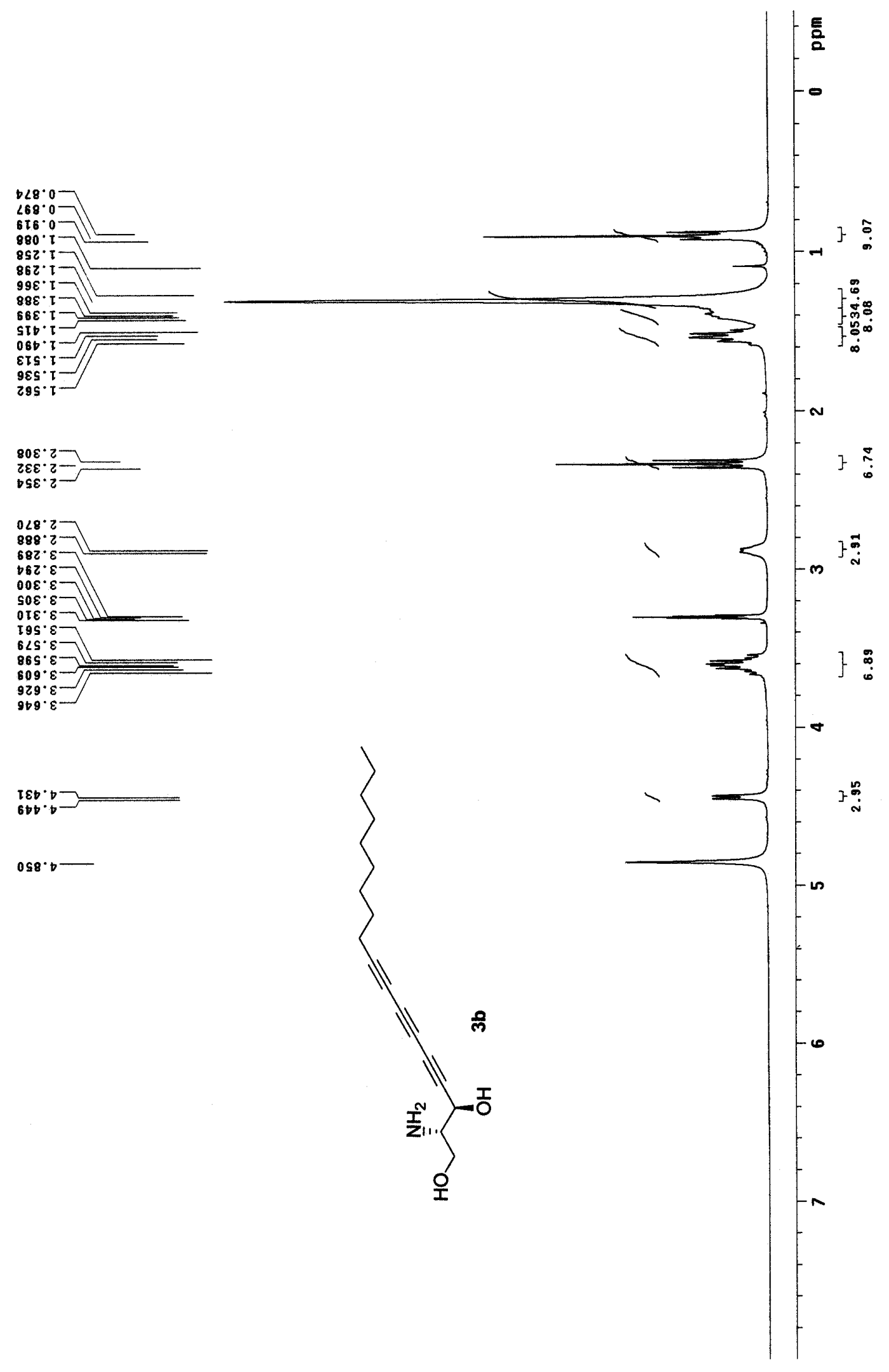



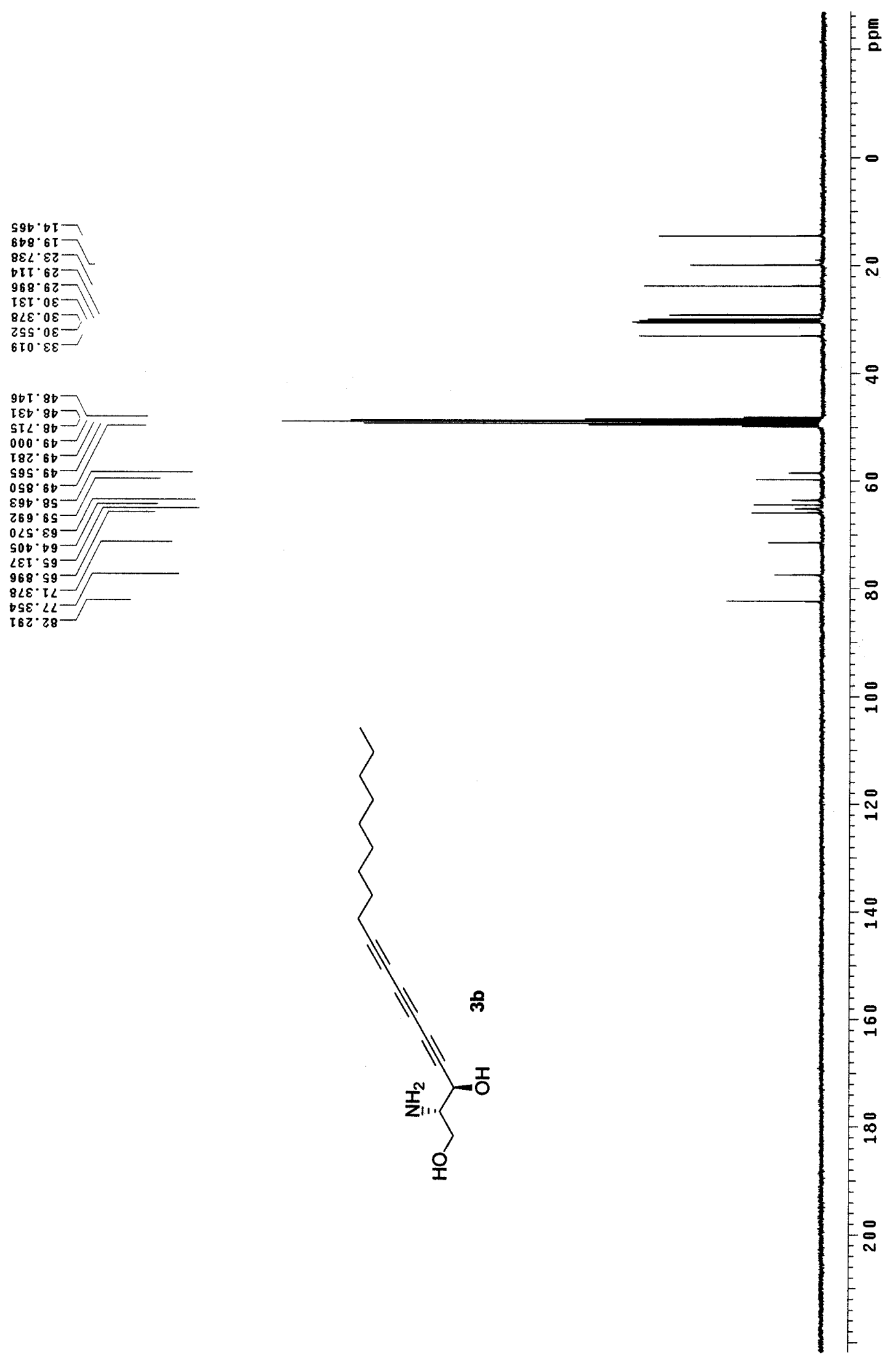

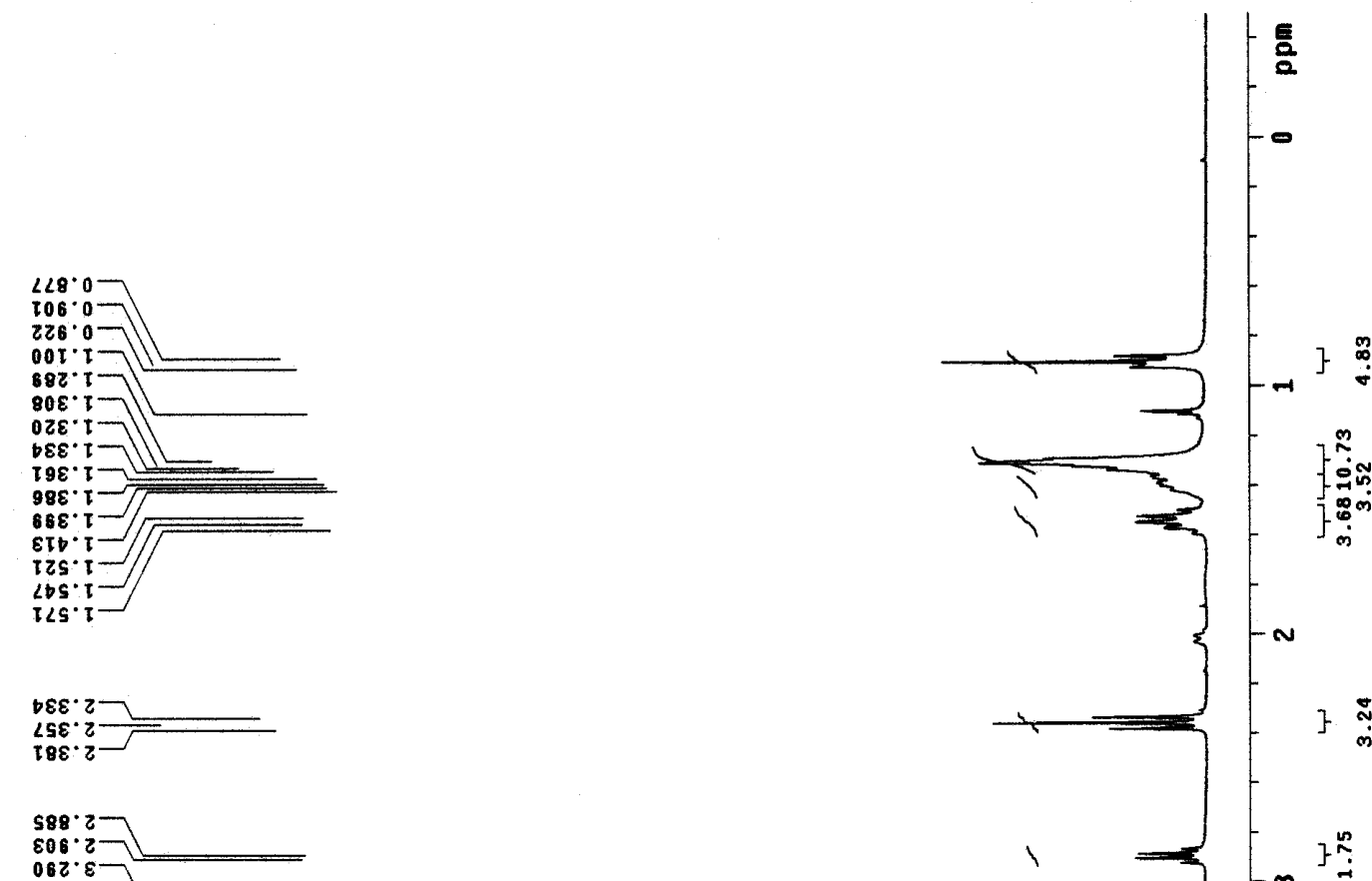

806.2
$062^{\circ} \cdot 8$

SBz. $\varepsilon$

$00 \varepsilon \cdot \varepsilon-$

$90 \varepsilon \cdot \varepsilon-1$

गद्ध

Sटह.

$085^{\circ} \varepsilon \square$

$009 \cdot$

$809^{\circ} \varepsilon$
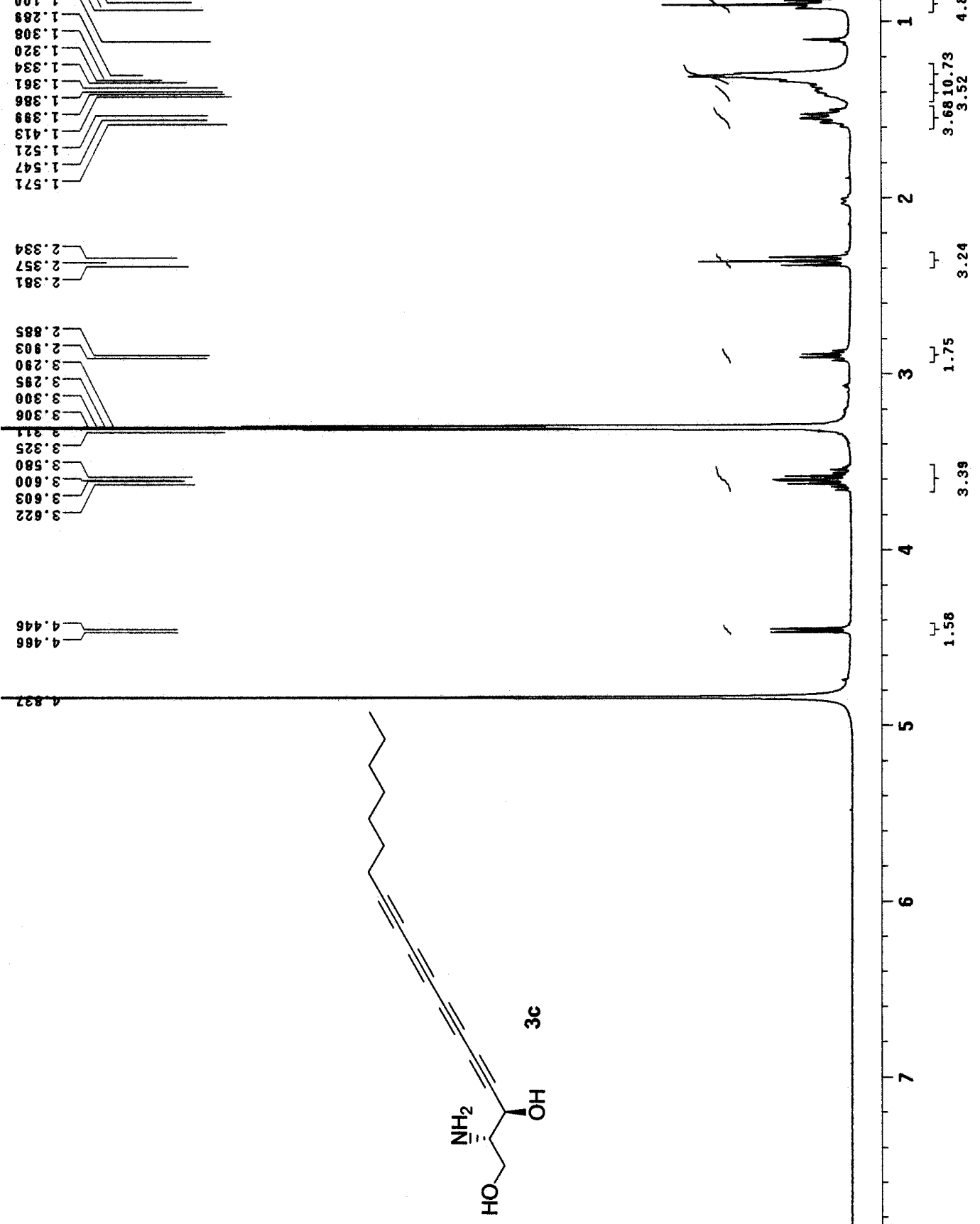

$=\{-\infty \stackrel{n}{-}$

f $\stackrel{0}{m}$
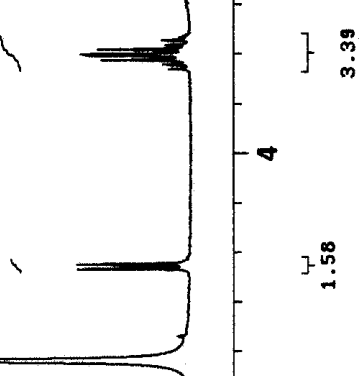

is

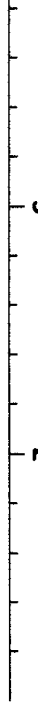




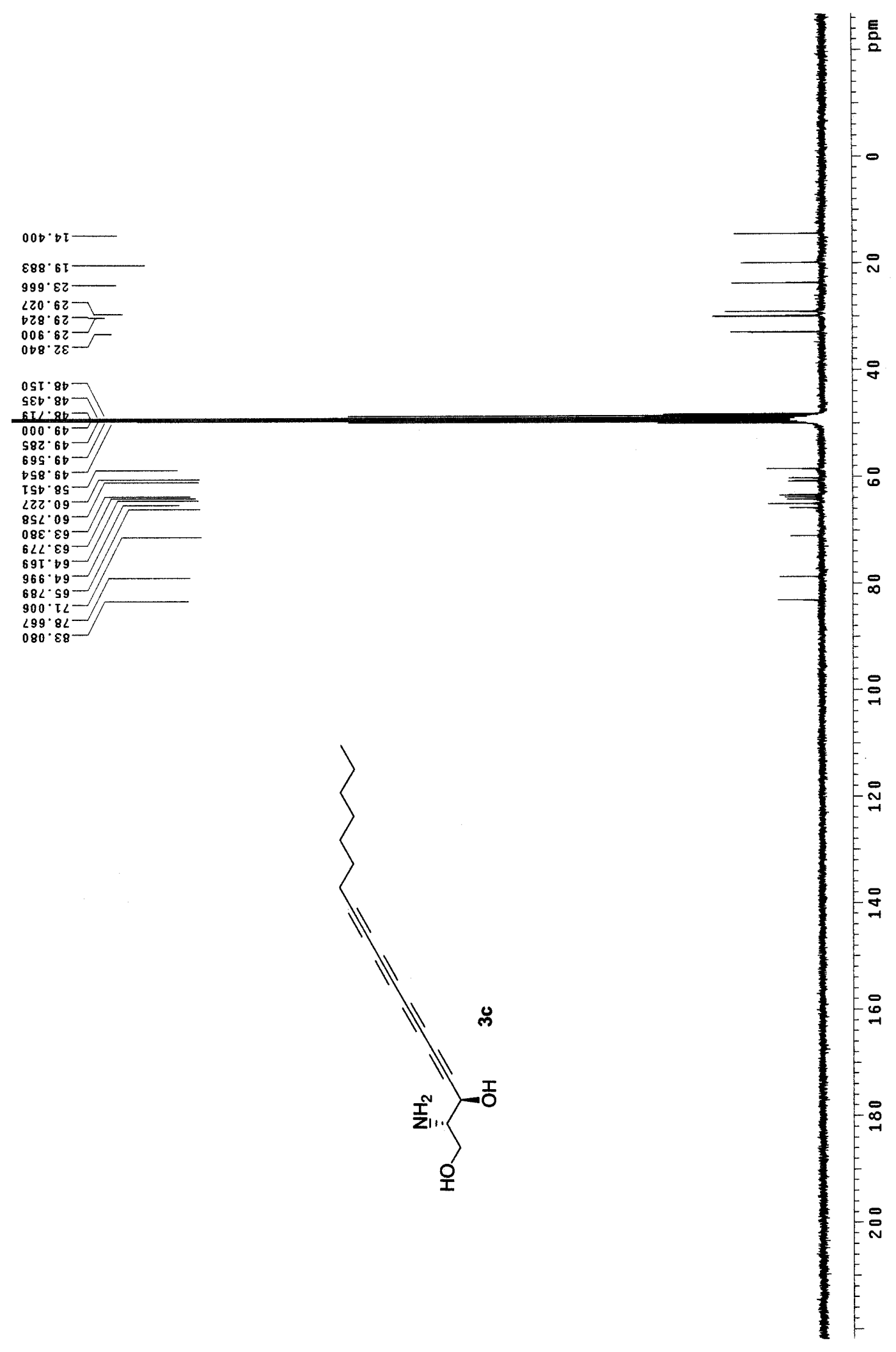



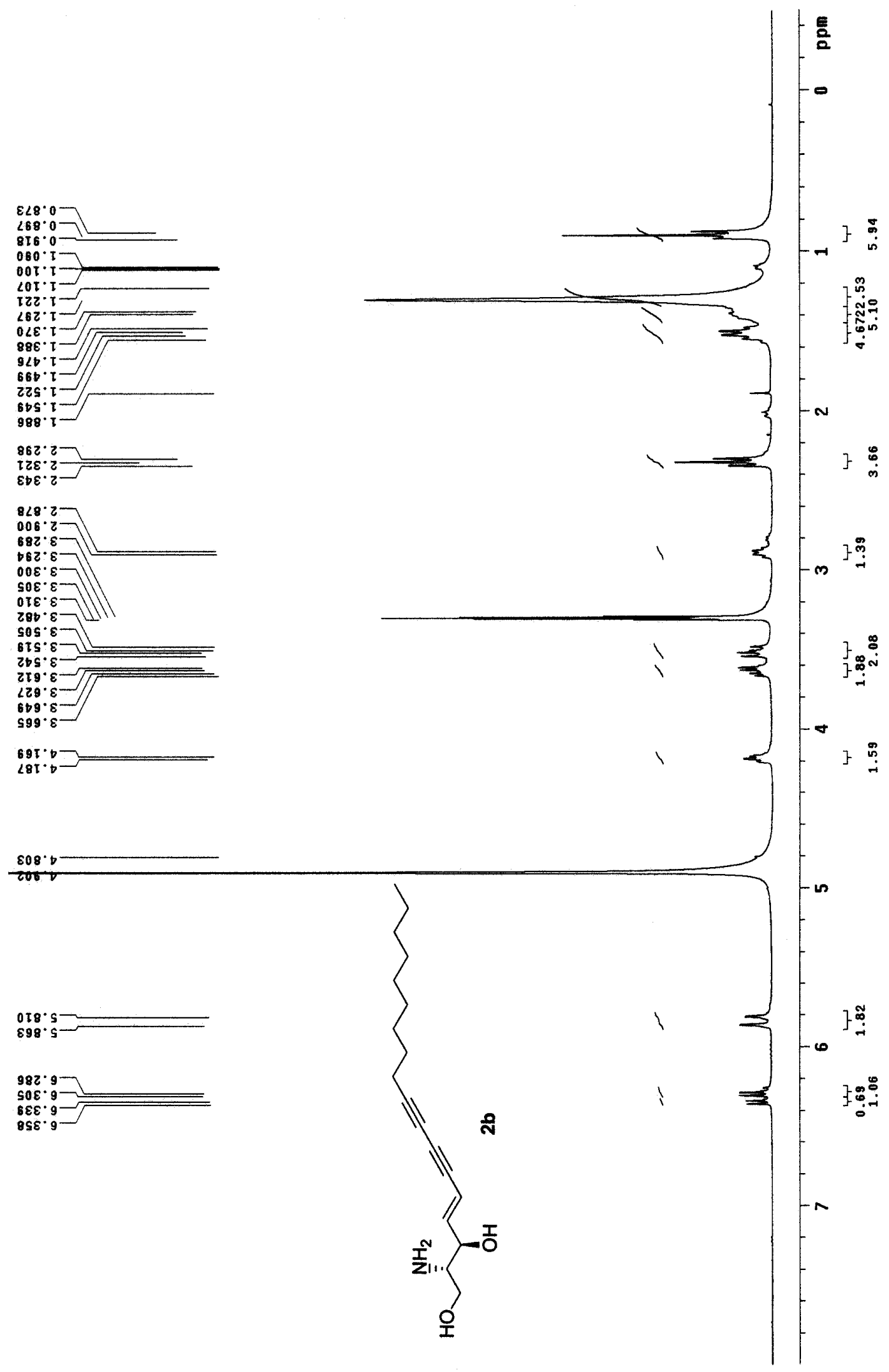


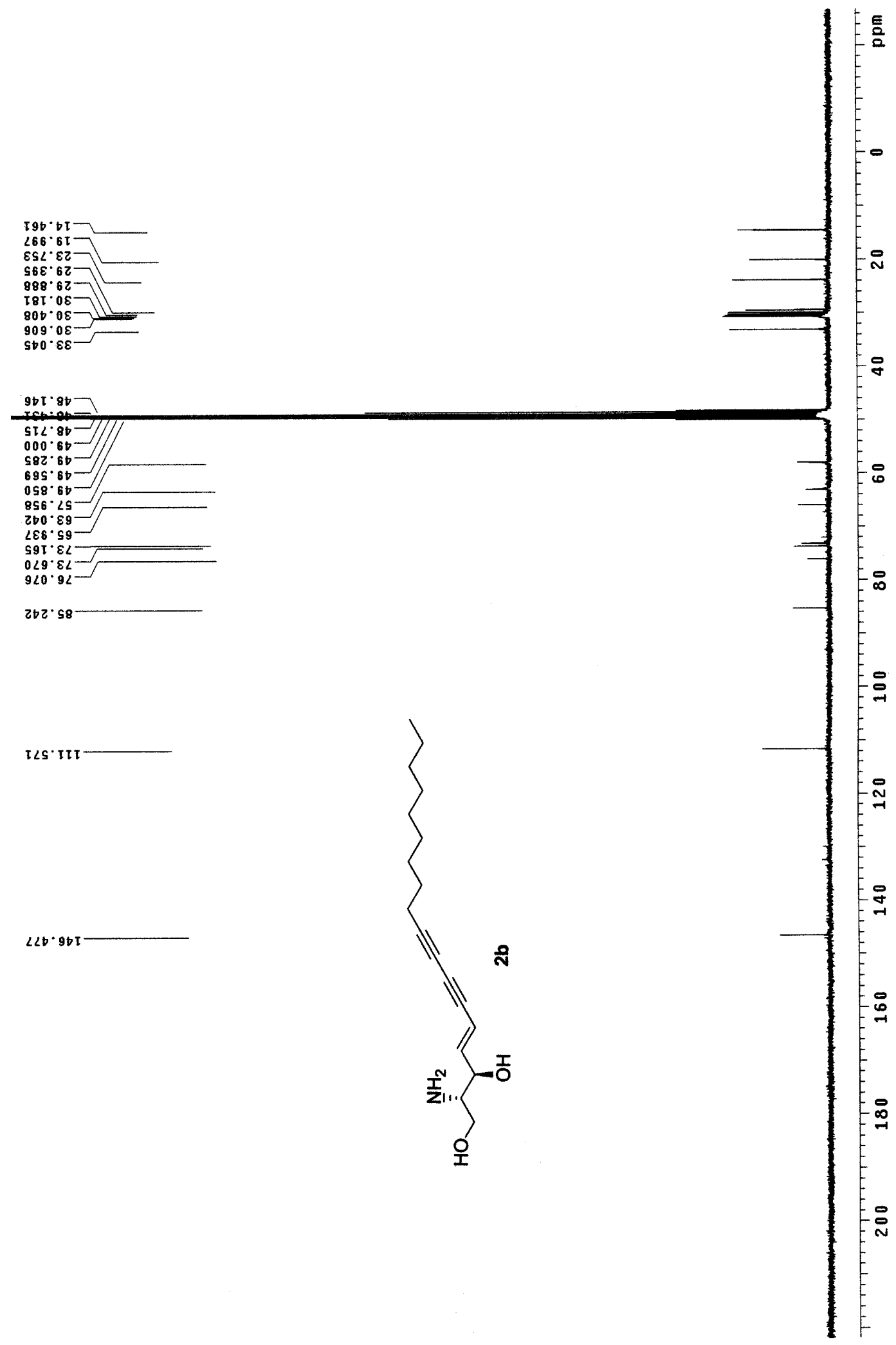



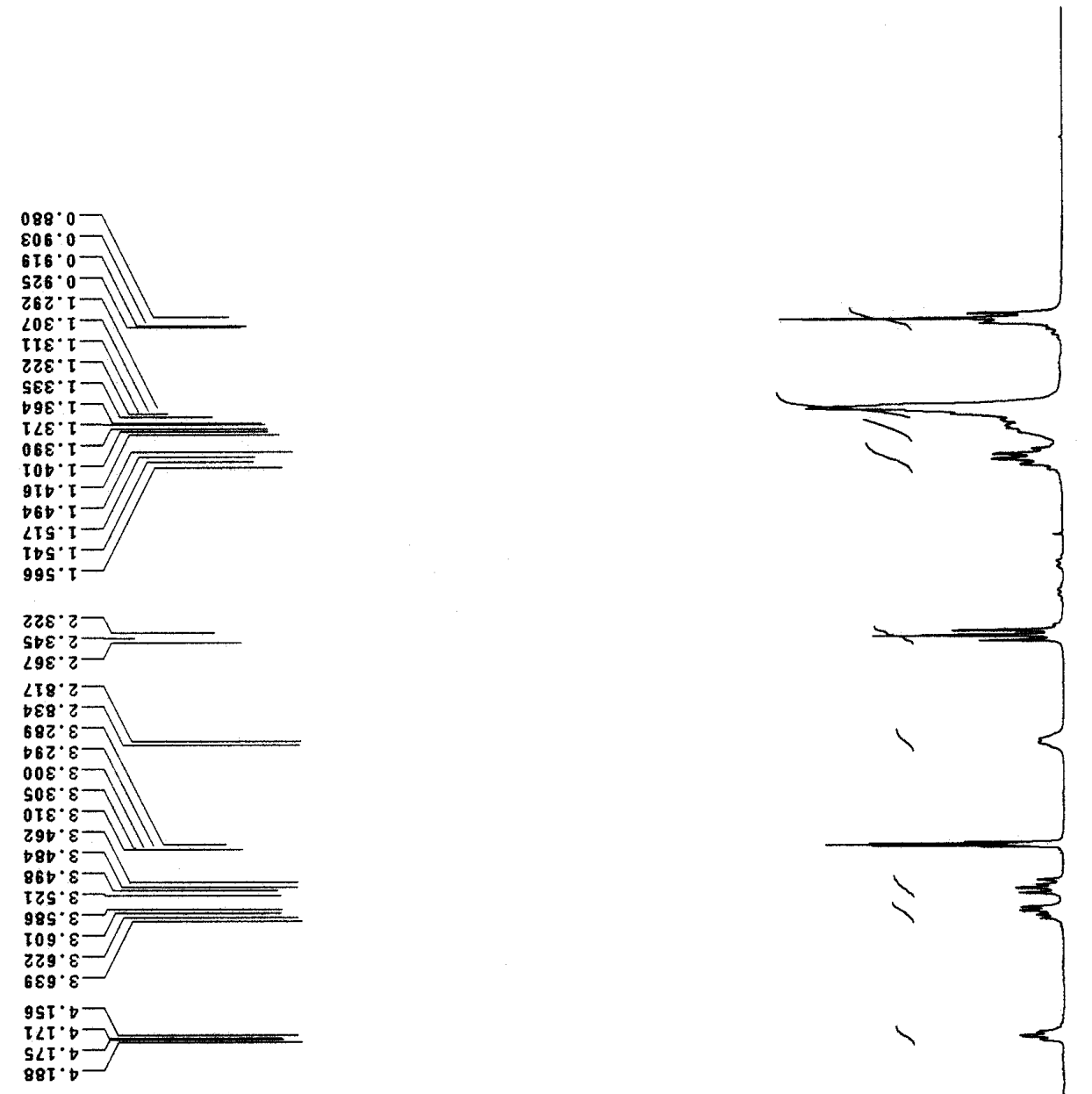

틍

$-$

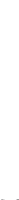

f $\stackrel{0}{:}$

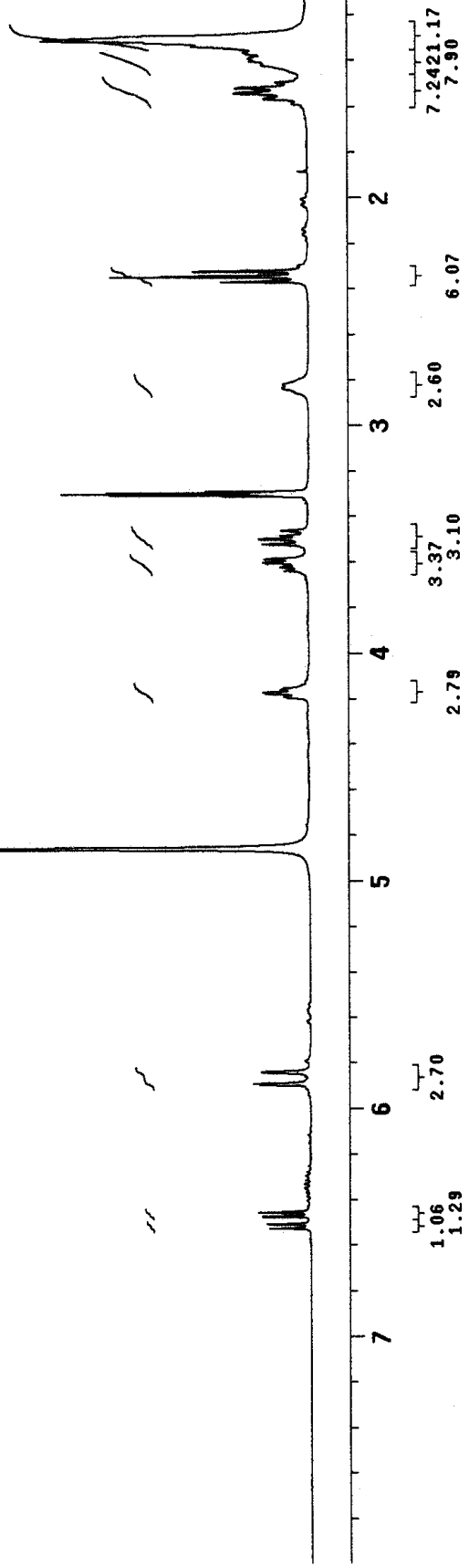

落

$\angle 98^{\circ} \cdot$

$988 \cdot \mathrm{s}$

$208.2=1$

$688^{\circ} \mathrm{s}-$

tsa. 9

$2 \angle 0 \cdot 9 \square \square$

$\angle 0 S \cdot 9$

$925 \cdot 9$

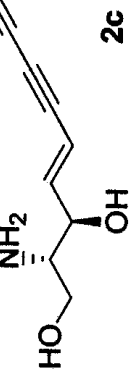




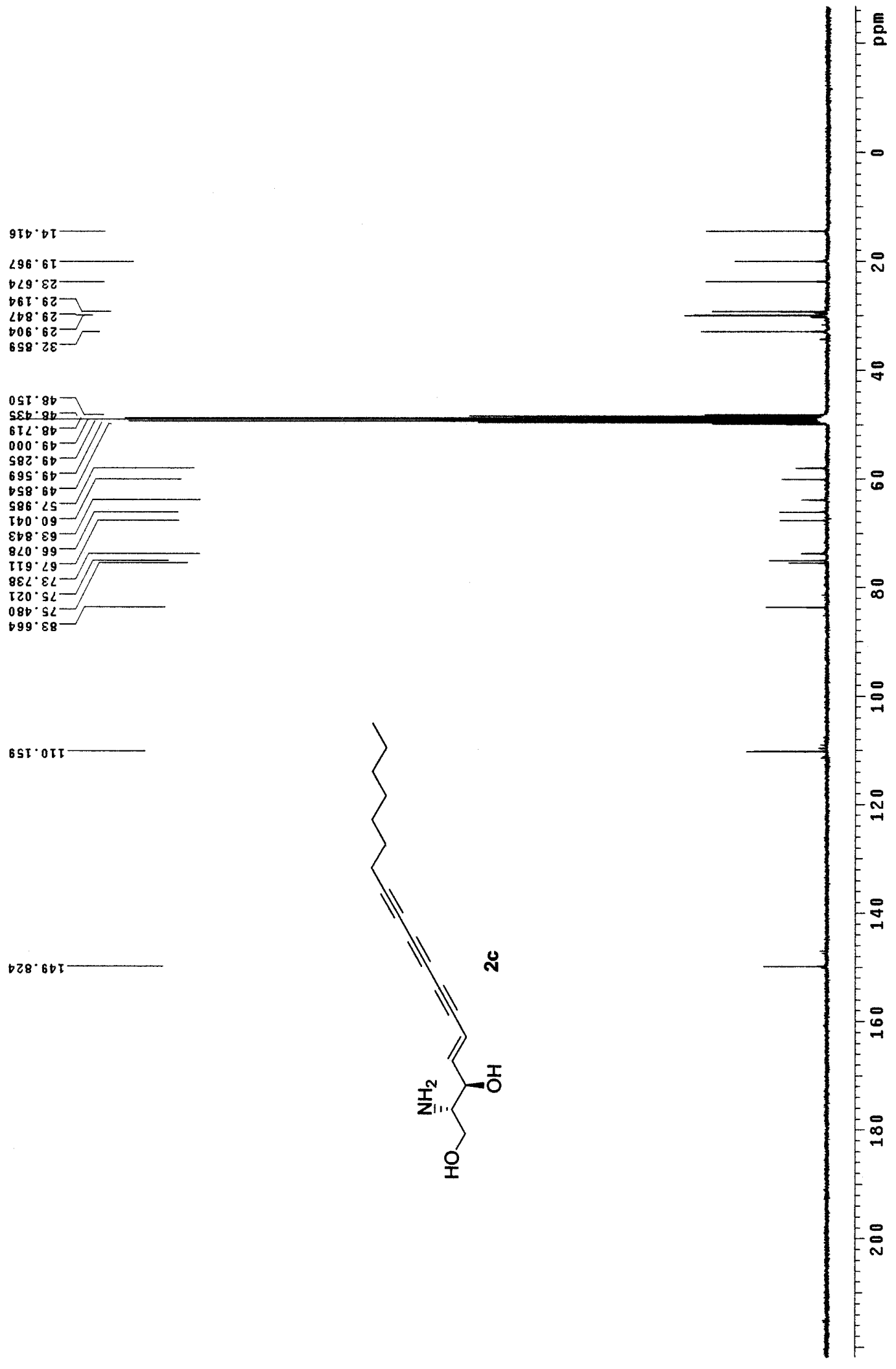

